Intersections

Canadian Journal of Music

Revue canadienne de musique
Intersections

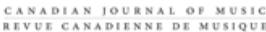

\title{
De la musique à l'architecture : le mystère des pans de verre " ondulatoires » du couvent de la tourette de le corbusier et Xenakis
}

\section{Elisavet Kiourtsoglou}

Volume 35, numéro 2, 2015

URI : https://id.erudit.org/iderudit/1043822ar

DOI : https://doi.org/10.7202/1043822ar

Aller au sommaire du numéro

Éditeur(s)

Canadian University Music Society / Société de musique des universités canadiennes

ISSN

1911-0146 (imprimé)

1918-512X (numérique)

Découvrir la revue

Citer cet article

Kiourtsoglou, E. (2015). De la musique à l'architecture : le mystère des pans de verre « ondulatoires » du couvent de la tourette de le corbusier et Xenakis. Intersections, 35(2), 75-117. https://doi.org/10.7202/1043822ar
Résumé de l'article

Les pans de verre « ondulatoires » du Couvent de la Tourette (1953-1961) une distribution irrégulière de pans de verre de longueurs graduellement variables - se présentent comme une nouvelle version de l'ancienne analogie entre musique et architecture ; c'est Iannis Xenakis, qui travaillait comme ingénieur et architecte dans l'atelier Le Corbusier, qui les a conçus en faisant appel à ses recherches musicales. Dans cet article, nous proposons une hypothèse pour déchiffrer leur conception et leur rapport avec son travail de compositeur, restés longtemps peu détaillés, en interprétant des éléments tirés des archives.
Copyright (C Canadian University Music Society / Société de musique des universités canadiennes, 2018
Ce document est protégé par la loi sur le droit d'auteur. L’utilisation des services d’Érudit (y compris la reproduction) est assujettie à sa politique d'utilisation que vous pouvez consulter en ligne.

https://apropos.erudit.org/fr/usagers/politique-dutilisation/ 


\title{
DE LA MUSIQUE À L'ARCHITECTURE : LE MYSTĖRE DES PANS DE VERRE "ONDULATOIRES" DU COUVENT DE LA TOURETTE DE LE CORBUSIER ET XENAKIS
}

\author{
Elisavet Kiourtsoglou
}

\section{INTRODUCTION}

En 1953, le père Couturier demande à Le Corbusier de construire un nouveau couvent pour la communauté dominicaine à Éveux-sur-l'Arbresle, près de Lyon. À cette époque, l'architecte suisse partageait son temps entre l'immense chantier de Chandigarh et différents projets d'habitations, tout en s'adonnant quotidiennement à la peinture. Pendant la même période, Iannis Xenakis, ingénieur depuis 1947 dans l'atelier de Le Corbusier, s'est senti prêt à franchir le cap et a demandé à son patron de lui confier un projet où il pourrait enfin s'exprimer comme architecte. Il aurait ainsi pour rôle d'associer des éléments du vocabulaire corbuséen (pilotis, toit-terrasse, rampe) avec le programme de construction - assez vaste - des frères dominicains $\left(\right.$ presque $5000 \mathrm{~m}^{2}$ ), tout en proposant des solutions aux problèmes du chantier. Mais sans doute sa contribution la plus connue est-elle la conception et la mise au point de pans de verres «ondulatoires» (appelés «musicales» par Le Corbusier) qui tapissent certaines façades du couvent.

À l'heure actuelle, tout ce dont on dispose sur les principes musicaux qui gèrent la nature et l'organisation de ces pans de verre se trouve dans le texte «Le monastère de la Tourette» que Xenakis a rédigé presque trente ans après la réalisation du couvent (Xenakis 1984 dans Kanach 2006, p. 105-119). Assez paradoxalement, pour quelqu'un qui a habitué ses lecteurs à des textes remplis de calculs et d'exemples concrets, cet article manque d'explications pour qu'on saisisse la mise en application de ces principes musicaux. Les chercheurs, tant musicologues qu'architectes, se sont heurtés tour à tour à ce manque d'éléments parlants' ${ }^{1}$.

La musicologue Anne-Sylvie Barthel-Calvet (2009, p. 9-51) est la première à émettre une hypothèse quantitative d'une relation entre les outils

1 Cf. Matossian 1981, p. 79; Ferro et al.1987, p. 91-94; Sterken 2003-2004, p. 127-133 \& p. 26o268; Kanach 2006, p. 73-121; Bridoux-Michel 2006, p. 224. 
mathématiques que Xenakis utilise pour sa musique et le travail qu'il mène pour la distribution des vitres sur les façades du couvent. Toutefois son hypothèse sur une distribution "probabiliste» des verres "ondulatoires» - en correspondance avec les compositions "stochastiques» de Xenakis de la même période comme les Pithoprakta (1955-56) - reste peu plausible, de l'aveu même de l'auteur.

Intriguée par ce "mystère», nous avons mené une enquête assez longue dans les archives de la Fondation Le Corbusier, ainsi que dans celles de Xenakis lui-même ${ }^{2}$. Nous avons été agréablement surprise de constater l'existence de multiples allers-retours, d'impasses ainsi que de changements d'orientation qui indiquent un vrai processus de germination des idées et non l'application d'une théorie «d'harmonie» quelconque, et encore moins la «traduction» directe du rythme d'une pièce musicale en architecture. L'hypothèse que nous avançons ici semble dissiper le mystère sur lequel repose l'organisation rythmique des pans « ondulatoires» du couvent. D'ailleurs, nous pouvons souligner aujourd'hui que l'analogie entre ces deux domaines, chez Xenakis, va dans les deux sens: on ne peut pas prétendre qu'il fait appel à ses recherches musicales lorsqu'il entreprend un projet d'architecte sans faire l'hypothèse inverse: Xenakis s'approprie la musique au moyen des mathématiques et des graphiques d'ingénieur et y met sa propre signature; il s'agit d'un univers créatif où les disciplines ne connaissent pas de frontières. Mais est-ce que cela n'est pas une condition préalable à toute forme de création?

\subsection{La "combinatoire» des Metastaseis et les façades intérieures du couvent}

Pour le Couvent de la Tourette, Xenakis fait appel à ses recherches musicales pour distribuer les pans de verre qui vitrent les façades intérieures et extérieures du couvent [fig.2 \& 1]. Les premières précèdent et préparent d'une certaine façon les deuxièmes, comme nous allons le voir, étant donné que c'est à partir de la distribution des éléments architecturaux sur les façades des étages qui donnent sur la cour intérieure que Xenakis affronte d'abord ce problème: il choisit d'y répondre en utilisant la combinatoire mathématique, dont les vertus et les faiblesses l'accompagnent aussi dans ses œuvres de la même époque (Metastaseis, 1953-54).

Comme il apparaîtra à l'analyse, les limitations que la combinatoire pose dans le cas des façades intérieures obligeront Xenakis à prendre une toute autre direction quand il sera question de façades extérieures, comme d'ailleurs c'était le cas pour sa musique pendant la même période: la combinatoire des Metastaseis va être remplacée par la théorie des probabilités dans les Pithoprakta. Dans l'architecture, cette tournure n'aura pas le même visage; Xenakis n'ayant pas encore pensé aux probabilités, pour les façades extérieures, il va mélanger certaines pratiques compositionnelles qui à cette époque n'existent encore

2 Archives de la Fondation Le Corbusier et Archives Xenakis, X(A) 2: La Tourette. Je tiens à remercier Mâkhi et Françoise Xenakis de m'avoir donnée accès aux archives Xenakis ainsi que Arnaud Dercelles et Delphine Studer de la Fondation Le Corbusier pour leur aide. Le présent article est une version abrégée du chapitre consacré à la Tourette et l'analogie du rythme dans Kiourtsoglou 2016, p. $82-160$. 


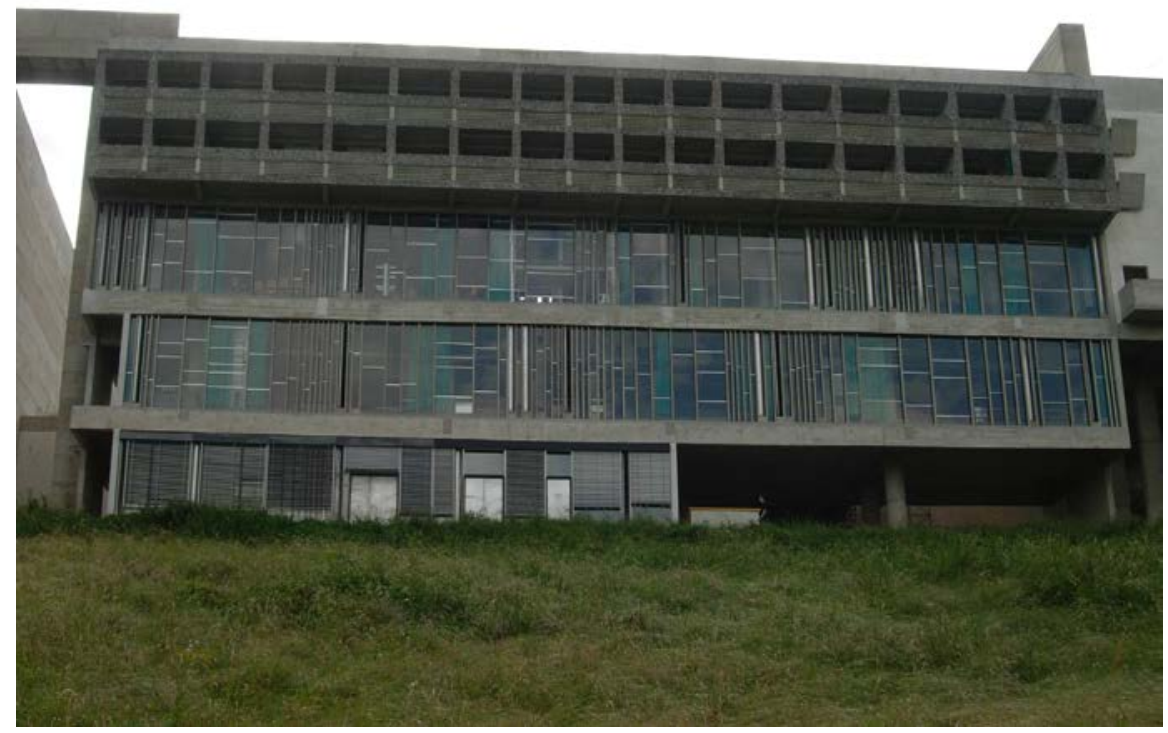

Figure 1. La facade exterieure ouest du Couvent de la Tourette (@ F.L.C. / Adagp, Paris, 2017) vitrée avec des pans de verre "ondulatoires". Image d'Elisavet Kiourtsoglou.

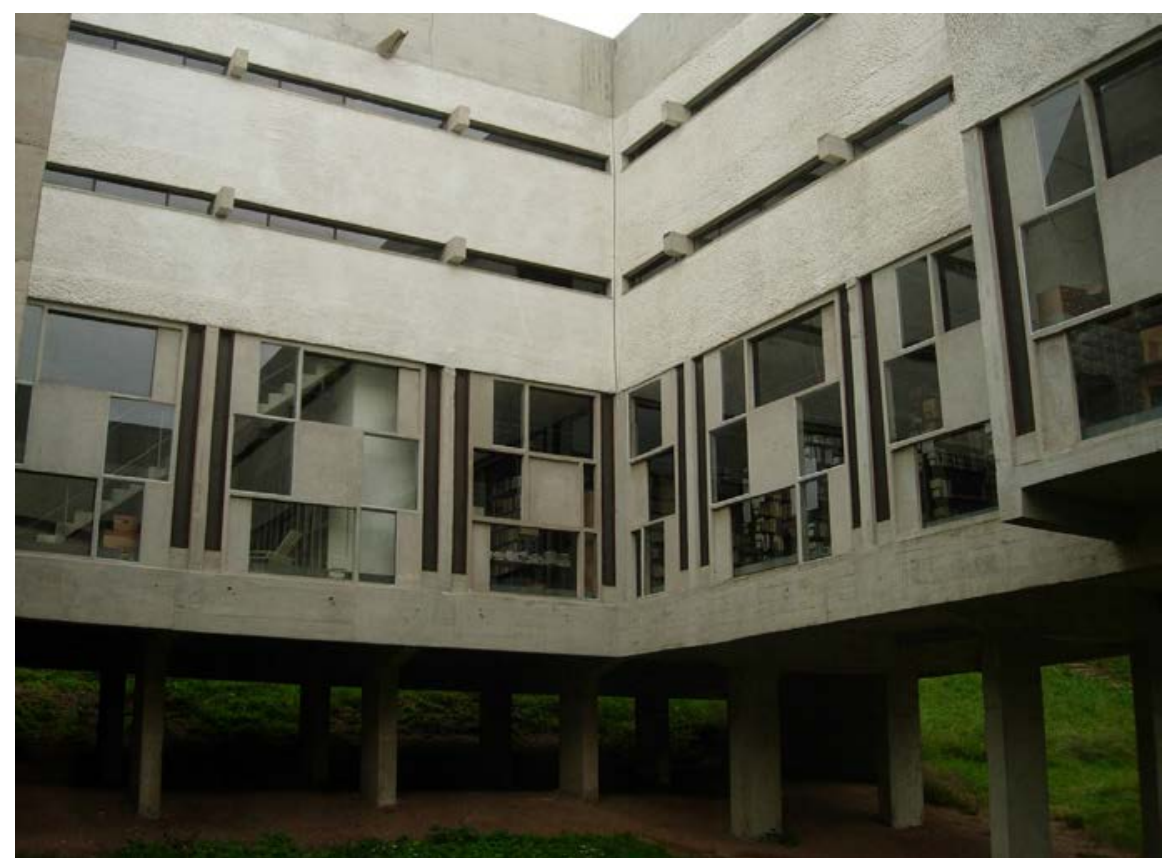

Figure 2. Une des facades intérieures du couvent de la Tourette (@ F.L.C. / Adagp, Paris, 2017) portante des pans de verre "combinatoires". Image d'Elisavet Kiourtsoglou. 
qu'à un stade embryonnaire; ici, l'architecture devient carrément le laboratoire où le compositeur, en touchant aux questions d'ordre spatial, avance dans sa recherche musicale. Retraçons donc d'abord l'histoire des pans de verre «combinatoires» des façades intérieures. ${ }^{3}$

Au printemps 1954, Xenakis commence à s'occuper des couloirs de circulation, qui donnent sur la cour intérieure du couvent et qui auraient dû être revêtus d'une forme de vitrage. Les plans $\mathrm{N}^{\circ} 2551$ (sans date) [fig.3] et $\mathrm{N}^{\circ} 2552$ (3-06-1954) [fig.4] montrent ses premières études pour la conception des panneaux, à l'intérieur desquels Xenakis mélange des morceaux de verre et des parties en béton ${ }^{4}$. À ce stade, les panneaux ont tous la même hauteur $(366 \mathrm{~cm})$ et la même longueur (probablement $376 \mathrm{~cm}$ ). Alors, comment distribuer les éléments en verre et les parties en béton à l'intérieur de ces panneaux? Xenakis se tourne vers ses compositions musicales, pour lesquelles se posent à la même époque des questions de répartition des éléments:

Je découvrais le vertige de la combinatoire d'éléments architecturaux après l'avoir expérimentée en musique. En effet, dans Metastaseis pour orchestre que je terminais à peu près à la même époque (1953-54), la partie médiane était bâtie sur une combinatoire d'intervalles mélodiques +-1,+$2,+-3,+-4,+-5,+-65$.[...] Je fis donc plusieurs études sur les trois façades, mais qui ne me satisfaisaient pas. J'avais choisi quatre éléments, a, b, c, d en section d'or et leurs vingt-quatre permutations que je disposais sur le déroulement des façades, comme une variation d'un même thème dans le temps. (Xenakis 1984, dans Kanach 2006, p. 112).

Dans le plan $\mathrm{N}^{\circ} 2551$ [fig.3], Xenakis choisit quatre longueurs de verre (a, b, c, d) tirées du Modulor ${ }^{6}$ et il établit leurs 24 permutations $\left(4 !=1^{*} 2^{*} 3^{*} 4=24\right)$. Dans ce plan, ces permutations passent exclusivement en haut de chaque panneau, tandis que le reste se remplit de béton. Les valeurs que nous arrivons à calculer sont $\mathrm{a}=70 \mathrm{~cm} \mathrm{~b}=140 \mathrm{~cm} \mathrm{c}=53 \mathrm{~cm} \mathrm{~d}=113 \mathrm{~cm}$. De surcroît, dans ce plan, Xena-

3 J'emploie le terme pans de verre «combinatoires» des façades intérieures pour les distinguer des pans de verres «ondulatoires» des façades extérieures, qui ont été nommés ainsi par Xenakis et Le Corbusier.

4 Tous les plans, sauf indication différente, renvoient à la collection de la Fondation Le Corbusier.

5 Exprimés en demi-tons : 1 demi-ton= +/-1.

6 Les deux séries du Modulor (Bleue et Rouge) ont été conçues par Le Corbusier pour être utilisées comme une grille des dimensions dans la reconstruction des habitats de l'après-guerre à une "échelle humaine». En effet, à partir d'un homme grand de 1,83m, qui avec le bras levé atteint 2,26m, et dont le nombril se trouve à $1,13 \mathrm{~m}$, deux séries de nombres se construisent autour de l'unité $(1,13)$, de son double $(2,26)$ et de deux sections dorées qui y sont ajoutées ou retranchées; la mesure 113 fournit la section d'or 70 et produit la série rouge $(4-6-10-16-27-43-70-113-183 \ldots)$; la mesure $226\left(2^{*} 113\right)$, son double, fournit la section d'or $140-86$ et construit la série bleue $(3-13-20-33-53-86-140-226 \ldots)$. Le quotient des deux nombres consécutifs est la meilleure approximation de la solution positive de l'équation $1+\sqrt{ }_{5} / 2$, dite «nombre d'or».

7 Barthel-Calvet 2009, p. 15. L'auteur propose comme valeurs $a=53 b=140 \quad c=33 d=86$. Nous avons redessiné le plan à l'échelle et les valeurs que nous y trouvons sont différentes : $a=70 \quad b=140 \quad c=53$ $\mathrm{d}=113$. Selon notre résultat, la longueur globale du panneau est d'une valeur de $376 \mathrm{~cm}$, tandis que selon le choix de Calvet, le panneau aurait dû mesurer $312 \mathrm{~cm}$. Pourtant la forme des panneaux sur les plans 


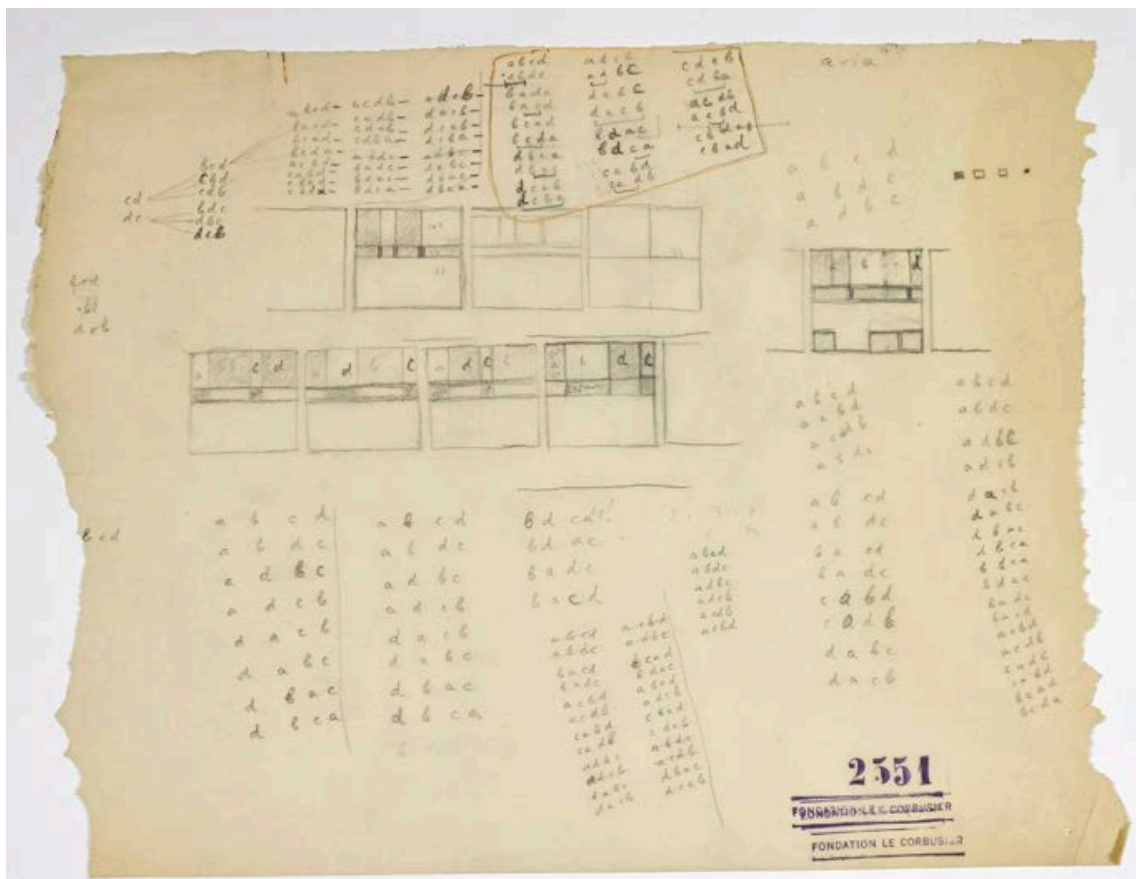

Figure 3. Croquis de Xenakis $\mathrm{N}^{\circ} 2551$ (sans date) avec les permutations mathématiques pour la distribution des pans de verre des façades intérieures du couvent, (C) F.L.C. I Adagp, Paris, 2017. Annoté en haut par Elisavet Kiourtsoglou.

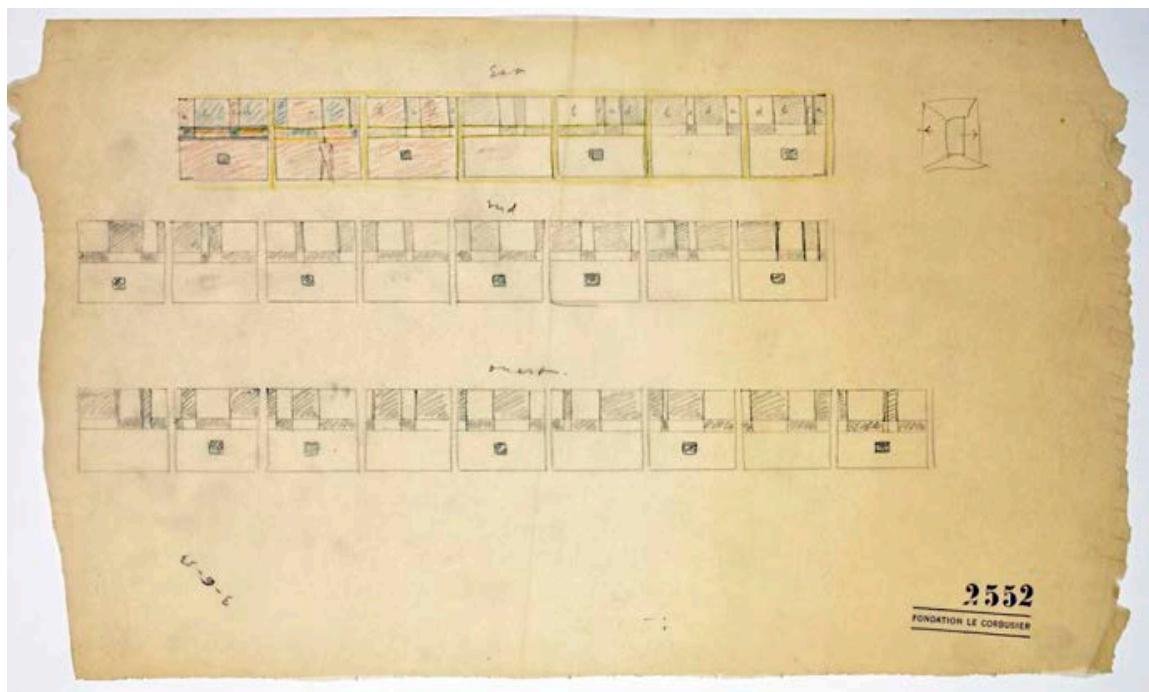

Figure 4. Croquis d'étude des pans de verre pour les trois façades intérieures par Xenakis, $\mathrm{N}^{\circ} 2552$, 3-06-1954, (c) F.L.C. / Adagp, Paris, 2017. 
kis indique les différentes façons de passer d'une permutation à l'autre; on y perçoit cinq configurations d'enchaînement. Certaines sont complètes avec la totalité des 24 permutations en place, d'autres contiennent seulement les premières permutations en place. Parmi ces cinq configurations, Xenakis en garde une seule qu'il va utiliser pour faire enchaîner les valeurs des pans de verres sur les panneaux, (Barthel Calvet 2009, p. 15). Nous soulignons en rouge sur ce plan la configuration choisie.

Le plan $\mathrm{N}^{\circ} 2552$ (3-6-1954) [fig.4] montre les trois façades (est, sud et ouest) des couloirs de circulation revêtues de ces panneaux. Ici, la configuration d'enchaînement finalement choisie avec ses 24 permutations s'étale tout au long des trois façades; les sept premières permutations se trouvent sur la façade est, les huit suivantes sur la façade sud et les neuf dernières sur la façade ouest.

Pourtant les architectes (Xenakis 1984, dans Kanach 2006, p. 112) se rendent compte très vite que «le jeu est très subtil pour qu'il saute aux yeux». En effet, sur toutes les façades, la partie haute $(183 \mathrm{~cm}$ sur $366 \mathrm{~cm}$ de hauteur) des panneaux a été réservée aux pans de verre, tandis que la partie basse contenait partout une surface lisse de béton. Vue de loin, l'alternance des différentes longueurs de verre passe presque inaperçue, étant donné que la partie basse de la case est toujours remplie de béton. Selon nous, il est assez plausible que ce soit pour cette raison que cette version soit restée dans les cartons ${ }^{8}$.

Du côté musical, à l'époque, Xenakis vient de finir la composition des $\mathrm{Me}$ tastaseis (1953-54) où il explore pleinement la liberté et la systématisation que la combinatoire lui offre. Dans son texte «Metastassis-Analyse», il détaille les procédés de permutations et les configurations d'enchaînements des séries de notes ou d'intervalles 9 . Ainsi, pour les mesures 104-150 des Metastaseis, il choisit quatre sons fixes (notes):

$\mathrm{A} 1=$ ré\# ré la la\#

$$
a \beta \gamma \delta
$$

qui donnent 24 permutations $\left(4 !=1^{*} 2^{*} 3^{*} 4=24\right)$. Xenakis les prend deux par deux et il forme 12 couples de permutations (A1-12) qu'il organise en deux colonnes et trois groupes [fig.5]. Chaque colonne contient deux couples Ai de permutations. Il détermine ensuite les lois d'enchaînement pour passer d'une colonne à l'autre et d'un groupe au suivant.

a plutôt l'allure d'un rectangle allongé, étant donné que sa hauteur est de $366 \mathrm{~cm}$. Dans tous les cas, ce qu'il est important de retenir ici, c'est la pratique des permutations et les différentes façons de les enchaîner. Les valeurs qui sont impliquées n'y changent rien.

8 Barthel-Calvet (Ibid.) suggère que ce projet n'a pas été retenu et qu'une version corbuséenne plus régulière et répétitive lui a été préférée. Selon elle, les alternances des permutations nécessitent une lecture horizontale des panneaux afin d'être perçues, ce qui est impossible à réaliser à partir d'un point fixe à l'intérieur de la cour du couvent. Nous nous permettons de nuancer cette observation car, comme on le verra plus loin, la proposition de Le Corbusier n'est pas très loin de celle que Xenakis essaie ici de mettre en place. Leur différence réside plutôt dans le fait que Le Corbusier utilise toute la surface du panneau pour distribuer les éléments tandis que Xenakis se limite au bandeau du haut.

9 Cf. Barthel-Calvet 2003, p. 129-187. 


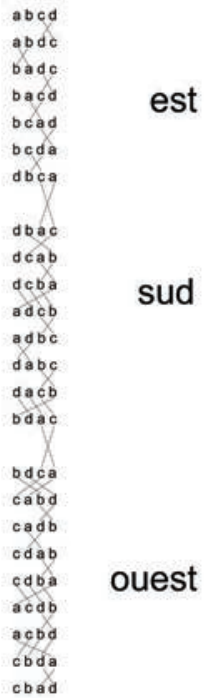

\begin{tabular}{|c|c|c|c|c|}
\hline$\vec{A}_{1}$ & $\begin{array}{l}a^{1} \beta^{5} \gamma^{1} \delta \\
a^{1} \beta^{4} \delta^{-1} \gamma\end{array}$ & $\overrightarrow{\mathrm{A}}_{3}$ & $\begin{array}{l}\beta a \delta \gamma \\
\beta a \gamma \delta\end{array}$ & \\
\hline $\overrightarrow{\mathrm{A}}_{2}$ & $\begin{array}{l}\gamma^{1} \delta^{4} \beta^{-1} a \\
\gamma \delta \alpha \beta\end{array}$ & $\overrightarrow{\mathrm{A}}_{4}$ & $\begin{array}{l}\delta \gamma \alpha \beta \\
\delta \gamma \beta \alpha\end{array}$ & Groupe 1 \\
\hline$\vec{A}_{5}$ & $\begin{array}{l}\alpha \delta \gamma \beta \\
a \delta \beta \gamma\end{array}$ & $\vec{A}_{7}$ & $\begin{array}{l}\delta a \beta \gamma \\
\delta a \gamma \beta\end{array}$ & Groupe 2 \\
\hline $\overrightarrow{\mathrm{A}}_{6}$ & $\begin{array}{l}\gamma \beta \delta \alpha \\
\gamma \beta \alpha \delta\end{array}$ & $\vec{A}_{8}$ & $\begin{array}{l}\beta \gamma \alpha \delta \\
\beta \gamma \delta \alpha\end{array}$ & \\
\hline $\overrightarrow{\mathrm{A}}_{9}$ & $\begin{array}{l}\alpha \gamma \beta \delta \\
a \gamma \delta \beta\end{array}$ & $\vec{A}_{11}$ & $\begin{array}{l}\gamma \alpha \delta \beta \\
\gamma \alpha \beta \delta\end{array}$ & Croune \\
\hline $\overrightarrow{\mathrm{A}}_{10}$ & $\begin{array}{l}\beta \delta \gamma \alpha \\
\beta \delta \alpha \gamma\end{array}$ & $\vec{A}_{12}$ & $\begin{array}{l}\delta \beta \alpha \gamma \\
\delta \beta \gamma \alpha\end{array}$ & Groupe 3 \\
\hline
\end{tabular}

Figure 5. A gauche: Permutations finalement choisies (du plan $\mathrm{N}^{\circ} 2551$ ) des quatre valeurs de pans de verre pour les façades intérieures du couvent, graphique établi par l'auteur (EK), en suivant le graphique de Barthel-Calvet, 2009, p. 38. À droite: Permutations de notes pour les mesures 104-150 de Métastaseis (à droite) comme données par Xenakis, publiée dans Barthel-Calvet, 2003, p. 162-163.

Dans chaque Ai la deuxième permutation change l'ordre des deux dernières notes.

Mais de A1 à A2 on va en rétrogradant la deuxième permutation de A1

De colonne à colonne: en rétrogradant la dernière permutation.

De groupe à groupe en choisissant les plus grands contrastes de permutations.

Résumé: Classification contrôlée des permutations par groupes de familles, les familles (colonnes) étant formées par des enchaînements de variantes secondaires. (Xenakis dans Barthel-Calvet 2003, p. 162-163. Nous soulignons).

Est-ce que Xenakis transpose exactement les 24 permutations de quatre notes musicales et leurs enchaînements à la distribution des quatre longueurs de verre sur les façades intérieures du couvent ? Barthel-Calvet (2009, p.15) examine une par une les permutations imaginées ou utilisées sur les façades du couvent et conclut que le compositeur garde certains principes de la combinatoire des Metastaseis, mais qu'il y introduit très vite des ruptures dans la systématisation que la combinatoire lui offre. Pour l'auteur, ceci corrobore un trait récurrent de la poétique xenakienne, à savoir l'introduction d'irrégularités une fois qu'un système a été défini. 
Pourquoi Xenakis ne s'est-il pas contenté de conserver les configurations d'enchaînement des Metastaseis ? Peut-être parce que sur les façades du couvent, l'architecte a besoin d'une différenciation plus nette entre les permutations des pans de verre; il doit changer plus souvent la place de toutes les valeurs lors du passage d'une permutation à l'autre. Sinon le jeu devient encore plus subtil pour être aperçu. C'est d'ailleurs la raison pour laquelle même cette dernière proposition n'a pas été retenue, le revêtement des façades intérieures prenant une tout autre direction à l'initiative de Le Corbusier, comme on le verra plus loin. D'ailleurs dans Metastaseis, les permutations des notes ont comme but d'engendrer différents intervalles auxquels Xenakis attache quatre "classes» de «durées différentielles», un nouvel élément rythmique, selon le nombre des demi-tons que les intervalles contiennent ${ }^{10}$. Barthel-Calvet (2003, p. 142-143) montre que les douze couples de permutations des hauteurs (A1-12) des Metastaseis s'attachent aux quatre classes de "durées différentielles» (a1, a2, a3, a4). Effectivement, nous pensons que le compositeur a tout intérêt à suivre des lois systématiques lors du passage d'une permutation à l'autre afin de préserver des liens de parenté entre les différents intervalles, et par conséquent à l'intérieur du «rythme» que les «durées différentielles» imposent. En revanche, dans le cas du couvent, l'architecte semble briser toute loi de périodicité et de systématisation pour l'enchaînement des permutations de quatre longueurs de verre dans la perspective de rendre plus lisible le jeu plastique qu' il entreprend. Le principe des permutations qui gouverne la pratique du compositeur ne se transpose pas mutatis mutandis quand il réapparait au sein de l'architecture. Les pans de verre «combinatoires» font appel à l'idée de la combinatoire et non pas à son application spécifique constatée dans les mesures des Metastaseis.

En revanche, Le Corbusier proposera à Xenakis d'utiliser toute la surface du panneau pour distribuer différents segments de béton armé et les différentes valeurs de pans de verre; le plan $\mathrm{N}^{\circ} 2549$ (6-11-1954) [fig.6] en atteste. Les petites esquisses à main levée du maître, en haut, guident les dessins que Xenakis (probablement) produit en bas. Sur ce plan, la façade intérieure ouest du troisième étage, celle des salles communes, est vitrée de façon homogène, avec la répétition de panneaux identiques. Au deuxième étage du réfectoire, les panneaux sont distribués avec des intervalles plus grands, tous les deux poteaux. Force est de souligner que Le Corbusier a déjà fait l'expérience de compartimenter un panneau selon les mesures du Modulor. Dans le premier tome du Modulor, l'architecte fait une longue référence au «Jeu des panneaux»; il s'agit de combinaisons possibles de rectangles en verre et de pans de menuiserie,

10 Le mécanisme de "la durée différentielle» a été décrit par Xenakis dans "METASTASSIS-Analyse» (1954) et a été détaillé par Barthel-Calvet 2003, p. 131-136. Selon la dernière, dans les structures monodiques, les durées dites «différentielles» coïncident avec les durées propres des sons. Par contre, dans le cadre polyphonique, la durée différentielle gère le décalage entre les durées des sons, qui appartiennent aux différentes lignes mélodiques, qui ont chacune une mesure différente $(5 / 16,4 / 16,3 / 8)$. Les durées propres des sons de chaque ligne sont rapportées à un module commun (la noire), qui permet l'algèbre élémentaire (soustraction) nécessaire aux calculs de la durée «différentielle». Ainsi une «durée différentielle» de valeur de 0,05 de la noire correspond à l'écart entre une double croche normale (égale à 0,25 noire) et une double croche de quintolet (égale à 0,20 noire). 


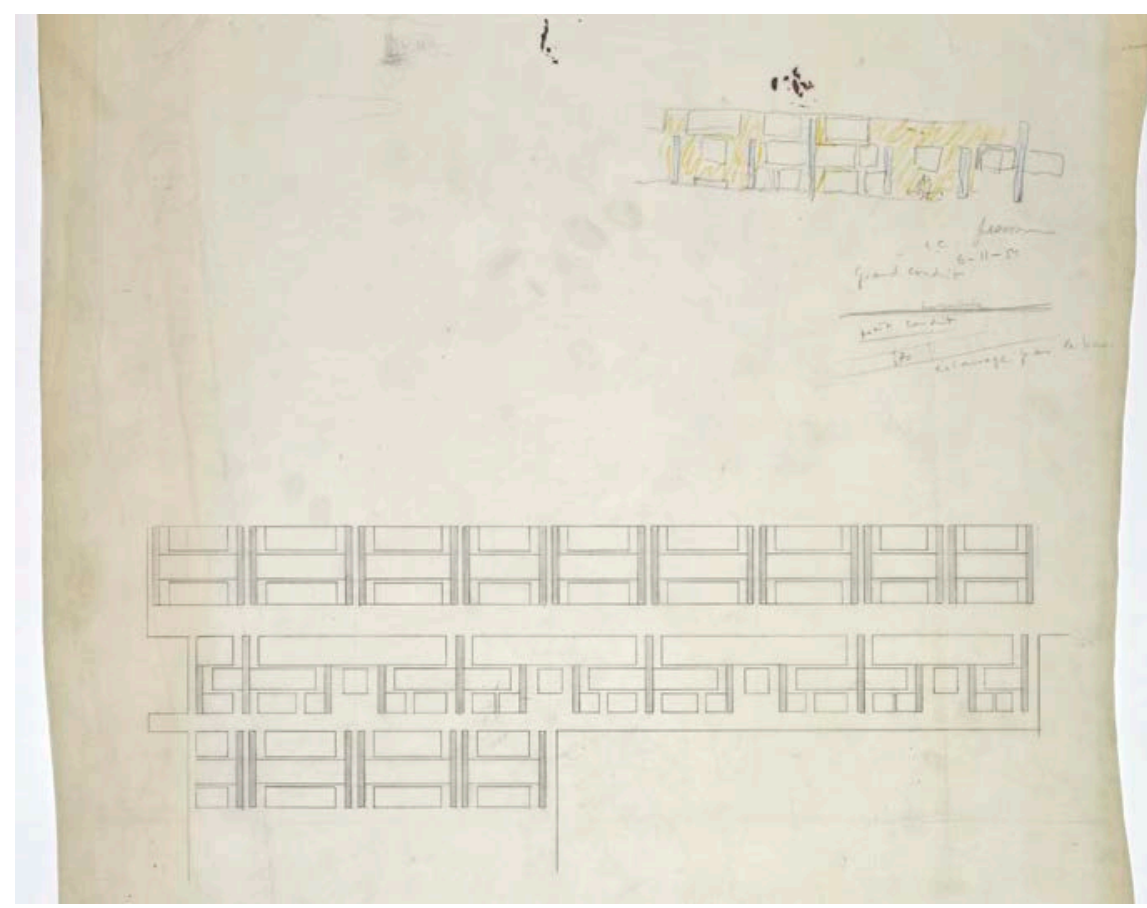

Figure 6. Elévation partielle de la façade intérieure $\mathrm{N}^{\circ} 2549$, aile ouest, 6-11-54 ㄷ F.L.C. I Adagp, Paris, 2017.

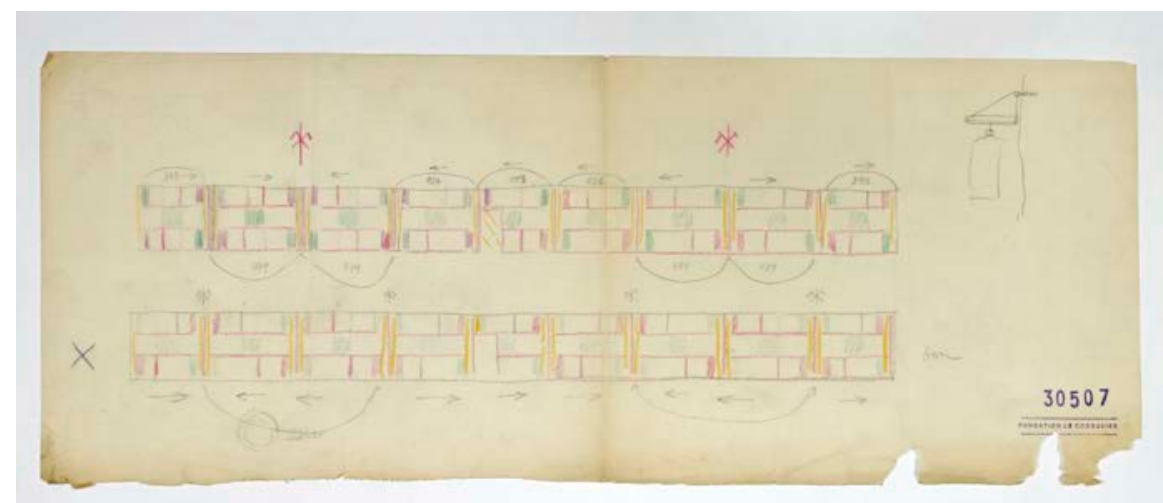

Figure 7. Croquis d'étude $\mathrm{N}^{\circ} 30507$ avec un «rythme» élémentaire pour la distribution des pans de verre des façades intérieures par Xenakis, sans date. (C) F.L.C. / Adagp, Paris, 2017.

dimensionnés à partir de Modulor, à l'intérieur d'un panneau (Le Corbusier 1949, p. 94-103).

Après ces discussions, une nouvelle variante des façades intérieures se présente ainsi dans les plans $\mathrm{N}^{\circ} 30507$ (sans date), $\mathrm{N}^{\circ} 30508$ (sans date) [fig.7] signées de la main de Xenakis. Les panneaux, toujours délimités entre deux poteaux, portent des pans de verre plus petits, qui alternent maintenant avec 
des parties en béton, grâce à l'indication de Le Corbusier. Dans ce plan, le crayon vert vaut pour le béton, le rose pour les vitres et le jaune pour les piliers qui délimitent les panneaux. Les panneaux mesurent toujours la même hauteur, mais à présent leur longueur varie. En effet, chaque longueur utilisée (393, 436, $479 \mathrm{~cm}$ ) est munie d'une disposition différente des parties en verre; les flèches au-dessus de chaque panneau indiquent si l'arrangement des vitres utilisé est la version originale $(\rightarrow)$ ou la version «rétrograde» $(\leftarrow)$ correspondant à cette longueur. Comme dans la musique sérielle, la version rétrograde se produit quand la version droite se lit à l'envers. La version rétrograde $(\leftarrow)$ d'un panneau de longueur $393 \mathrm{~cm}$ mesure toujours $393 \mathrm{~cm}$, mais les vitres sont distribuées à l'envers, de façon symétrique autour d'un axe vertical. Dans le plan $\mathrm{N}^{\circ} 30507$ [fig.7], Xenakis met en place deux «rythmes» élémentaires pour ces façades intérieures, fondés sur la succession des versions (originales et rétrogrades) des panneaux de longueurs différentes $(393,436,479 \mathrm{~cm})$. Nous les transcrivons ici:
a) $\rightarrow 393, \rightarrow 479, \leftarrow 479, \leftarrow 436, \leftarrow 393, \leftarrow 436, \leftarrow 479, \rightarrow 479, \rightarrow 393$
b) $\rightarrow 393, \leftarrow 479, \leftarrow 479, \rightarrow 436, \rightarrow 393, \rightarrow 436, \leftarrow 479, \leftarrow 479, \rightarrow 393$

Xenakis choisit le deuxième rang qu'il qualifie de «bon». En effet, pour le deuxième rythme (b), qui est finalement retenu, l'architecte rompt avec toute forme de symétrie apparue au sein du premier rythme (a); les versions rétrogrades n'apparaissent pas en miroir avec leurs versions originales puisque Xenakis met bout à bout deux versions rétrogrades (ici en italique). Cela donne l'impression d'une rupture, tout en utilisant exactement les mêmes panneaux. Xenakis passe ainsi des permutations, qui concernent des longueurs de verres solitaires - comme nous l'avons vu avec les 24 permutations des quatre longueurs de verre - aux permutations des panneaux qui contiennent des ensembles de pans de verre.

Il est très intéressant de souligner que dans Metastaseis, pour les mesures 150-174, Xenakis a utilisé 17 permutations parallèles de deux tronçons (B et $\beta$ ) d'une série des notes $S$ (B1-17 \& $\beta$ 1-17), qu'il superpose sous leur forme droite $\rightarrow$ ou rétrograde $(\leftarrow)$, formant une structure en miroir: l'ordre croissant des permutations (B1-17 \& $\left.\beta_{1-17}\right)$ fait respectivement face à l'ordre décroissant

\section{On ${ }^{14}$ superpose quatre fois les $\vec{B}_{i}$ et les $\vec{\beta}_{i}$ comme ci-dessous :}

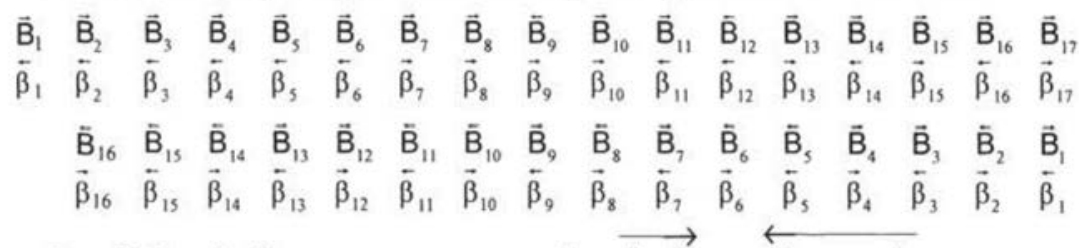

Les flèches indiquent une permutation droite ou rétrograde.

Figure 8. La superposition des 34 permutations «parallèles» des deux tronçons $B 1 \& \beta_{1}$ avec leurs formes droites et rétrogrades par Xenakis, publié dans Barthel-Calvet, 2003, p. 166. Avec l'aimable autorisation de Barthel-Calvet. 
(B17-1 \& b17-1) de ces mêmes permutations (Barthel-Calvet 2003, p. 147-150 et p. 164-167 ) [fig.8]. Le compositeur s'intéresse au nombre des demi-tons $(-/+1=1$ demi-ton) qui correspond à chaque intervalle entre les notes des deux tronçons. Ici chacune des 17 permutations $\mathrm{B}_{1-17} \& \beta_{1-17}$ apparaît sous sa forme droite ou rétrograde, selon que les intervalles entre les notes sont lus respectivement de gauche à droite ou de droite à gauche. Dans le cas de l'architecture, la symétrie entre la forme rétrograde et droite d'un panneau suit la définition géométrique au pied de la lettre: correspondance par rapport à un axe vertical: les trois types de panneaux $(393,436,479 \mathrm{~cm})$ se montrent sur leur forme "droite» ou «rétrograde», selon que les pans de verre sont disposés respectivement de gauche à droite ou de droite à gauche.

Le rythme élémentaire que Xenakis a qualifié de «bon» dans le plan $\mathrm{N}^{\circ} 30507$ sera assez proche de celui finalement retenu et utilisé dans $\mathrm{N}^{\circ} 1034$ (19-11-1954), plan récapitulatif où toutes les façades du couvent (intérieures et extérieures) contiennent des agencements de béton et de verre - en forme de « $\mathrm{H}$ » et de « $\mathrm{Z}$ » - à l'intérieur de chaque panneau. Toujours sur ce même plan, les façades extérieures du couvent (aile ouest, sud, est) sont aussi vitrées de pans de verre "combinatoires». Cela veut dire que fin 1954, les deux étages des salles communes et du réfectoire (qui portent aujourd'hui des pans de verre "ondulatoires») portaient des pans de verre "combinatoires".

\subsection{Les pans de verre "ondulatoires» et les façades extérieures}

C'est seulement au printemps 1955 que les architectes vont s'occuper à nouveau des façades extérieures du couvent. Le Corbusier, de retour du Chandigarh, fait découvrir à Xenakis un croquis de Pierre Jeanneret concernant l'astuce que les maçons indiens ont trouvée pour économiser les chutes de verre. Il s'agissait d'«un vitrage fait des montants verticaux espacés régulièrement, et contenant des vitres de hauteurs variables [les chutes de verre] mais de largeurs identiques, empilées «l'une sur l'autre sur toute la hauteur de l'étage» (Xenakis 1984, dans Kanach 2006, p. 113).

Cette première idée concerne donc la disposition verticale de différents morceaux de verre; Xenakis va aussi appliquer ce principe d'empilage horizontalement, tout au long de la façade. Selon Le Corbusier, pour distribuer horizontalement des pans de verres, deux solutions sont adéquates:

La première, la plus banale, consiste en une disposition à distances égales des membrures ${ }^{11}$. La seconde, plus savante, consiste à créer des motifs rythmiques en répartissant les membrures à des distances variables suivant une progression arithmétique. Ces deux solutions sont statiques. (Le Corbusier 1955, p. 339-340. Nous soulignons).

Optant pour une troisième solution «dynamique», celle où les longueurs de verre puisent leurs valeurs dans les deux séries du Modulor, Xenakis se trouve de nouveau devant un problème de distribution linéaire, comme quelques mois auparavant avec les pans de verre «combinatoires»:

11 Comme les maçons indiens faisaient selon le croquis de Pierre Jeanneret. 
La hantise de la combinatoire ne m’avait pas abandonnée, mais d'emblée je choisis plusieurs distances en sections d'Or tirées du Modulor. Mon problème était comment distribuer ces montants de béton dont je définissais la section standard, avec feuillures pour recevoir les vitres; comment les distribuer sur les façades. Autrement dit, comment distribuer des points sur une droite. (Xenakis 1984, dans Kanach 2006, p. 113).

La question ainsi posée, selon Xenakis, deux réponses se présentaient: soit choisir ces points en suivant une périodicité stricte, en créant des répétitions et des récurrences apparentes; soit ne pas doter cette répartition de périodicité, en distribuant aléatoirement les éléments. Toutefois, selon lui, le temps de l'architecture «stochastique» n'était pas encore venu

Mais, à l'époque, je ne faisais qu'entrevoir une musique stochastique (que j'allais inventer l'année suivante) et par conséquent je passais à côté de cette solution en architecture. Je le regrette beaucoup, car c'était une occasion unique d'introduire les probabilités en architecture. (Xenakis 1984, dans Kanach 2006, p. 113).

Xenakis reste donc dans la périodicité stricte, qu’il a appliquée au traitement des façades intérieures, comme nous l'avons vu dans la section précédente. Pour augmenter la complexité du jeu, il choisit maintenant dix distances, tirées des séries bleue et rouge du Modulor, qu'il essaie de distribuer sur la façade en se servant de leurs permutations ${ }^{12}$. Mais, s'il est encore facile de contrôler les 24 permutations de quatre longueurs de verre, il est impossible de le faire avec une dizaine de longueurs $\left(1^{*} 2^{*} 3 . .^{*} 10=10 !=3.628 .800\right.$ arrangements possibles $)$. En restant dans la périodicité stricte et les permutations, Xenakis va très vite se heurter à un très grand nombre de possibilités pour le placement des éléments. Il fait marche arrière et examine le même problème dans ses recherches musicales :

J'avais déjà traité ce problème en musique en créant des points (tops) sur une bande magnétique à des distances en section d'Or, et même une partition inédite pour percussion. Le critère était celui des fluctuations des densités des points (tops) sur la droite (temps). (Xenakis 1984, dans Kanach, 2006, p. 113).

En fait, Xenakis avait remarqué que l'arrêt ou le démarrage d'un enregistrement sur un magnétophone, qu'il venait d'acheter, laissait un «bip» sonore. Selon Ferro et al. (1987, p. 90) "c'est en jouant sur cette particularité qu'il créa, sur la bande, un premier rythme "ondulatoire», espaçant les interruptions de façon plus ou moins rapide, créant des crescendos et des decrescendos.»

Ainsi, au lieu de "contrôler» la répartition des dix éléments sur la façade, Xenakis va tenter de créer des fluctuations de la concentration des éléments des verres. Dans ce sens, il va juxtaposer et opposer des parties denses et raréfiées. Dans cette analogie, une grande densité correspond à un grand nombre de petites distances de verre (ou un grand nombre de membrures en béton qui 
les séparent). La répartition serait ainsi fondée sur un jugement statistique élémentaire: opposer des parties denses à des parties raréfiées et créer des fluctuations qui ressemblent effectivement aux « ondulations des milieux élastiques ${ }^{13}$ ». Le contrôle maintenant est plus aisé, étant donné qu'il y a moins d'éléments à prendre en compte. Mais il y a encore trois autres problèmes qui surgissent:

Donc la solution est de juxtaposer des parties denses (beaucoup de montants en béton armé) à des raréfiées. Ici, naturellement, il faut définir les degrés de densité et leurs longueurs (durées). Mais, de plus, un autre problème surgit, c'est celui du passage d'une densité à une autre, en progression continue, ou brutalement, par saccades. Le problème de la continuité dans la transition, ainsi que sa vitesse ou sa forme, jouent des rôles fondamentaux en esthétique musicale ou en arts plastiques et en architecture. (Xenakis, 1984 dans Kanach 2006, p. 116, nous soulignons).

Pourtant, le texte de Xenakis s'arrête ici et ne donne aucun exemple concret pour comprendre comment l'architecte a résolu pratiquement ces problèmes. Nous savons que toutes ces préoccupations vont faire naître ce que nous désignons aujourd'hui par pans de verre "ondulatoires». Différentes distances de verre, calées entre des membrures de béton, et réparties sur une façade sans poteaux ni murs porteurs de façon à ce qu'elles engendrent une sorte d'ondulation. Les façades extérieures du couvent, ainsi que les conduits en croix de la cour intérieure, seront revêtus de ce type de vitrage. Les pans de verre "ondulatoires» seront aussi appliqués dans un nombre important de projets de l'atelier Le Corbusier, même après le départ de Xenakis (Maison du Brésil/Cité Universitaire de Paris, Maison de la Culture à Firminy). Dans le souci de préserver le caractère et les principes qui gèrent leur application auprès de dessinateurs et d'architectes de l'atelier, Le Corbusier, pendant l'été 1955, va déposer un brevet pour les pans de verre dénommés «ondulatoires ${ }^{14}$ ». Et c'est à partir de là que l'on pourrait déplier leur histoire et s'approcher du mystère qui les accompagne.

Dans le texte (12-07-1955) qui accompagne le brevet, à côté du texte de l'architecte suisse, on trouve une petite note de la main de Xenakis qui dénombre les principes qui gèrent la distribution des éléments.

La disposition des éléments dans les ouvertures est en principe libre. N'importe quelle série de distances est valable, depuis la distance unique jusqu'à des progressions logarithmiques de distances en passant par des permutations de quelques longueurs quelconques. Toutefois le caractère dominant du système consiste dans l'emploi de quelques gammes de distances répétées sous forme d'ondes. Un tableau montre le principe dans les ondes de forme $\mathrm{A}^{\mathrm{m}} / \mathrm{n}$ et $\mathrm{B}^{\mathrm{m}} / \mathrm{n}$ dont les distances sont tirées des séries bleue et rouge du Modulor. Les ondes de forme $\mathrm{C}$ sont constituées par des juxtapositions de fragments des $A^{\mathrm{m}} / n$ et $B^{\mathrm{m}} / n$. Enfin, les formes $\mathrm{D}$ sont engendrées par des oppositions de dispositions denses ou raréfiées des éléments. (Xenakis 1955b, nous soulignons).

13 Le Corbusier 1955, p. 340.

14 Le texte du brevet de Le Corbusier est reproduit dans Kanach 2006, p. 76-77. 


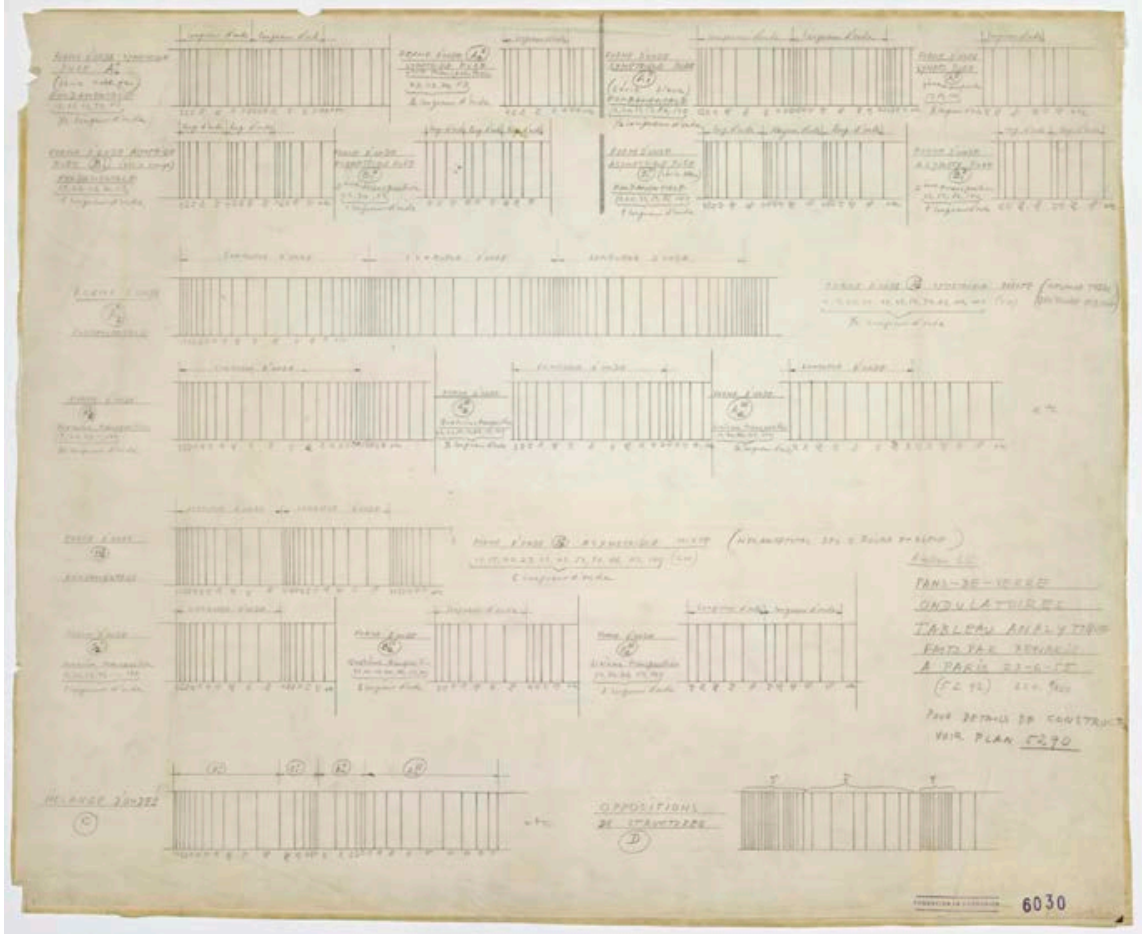

Figure 9. Tableau qui accompagne le brevet de pans de verre «ondulatoires» par Xenakis, N6030, 23-06-1955, (C) F.L.C. / Adagp, Paris, 2017.

Xenakis ici renvoie au tableau $\mathrm{N}^{\circ} 6030$ (23-06-1955) [fig.9] qui expose les différentes façons de faire succéder les dix distances tirées des séries Rouge et Bleue du Modulor. Il forme ainsi des «ondes», en réalité des séries de longueurs en ordre graduellement croissant ou décroissant, qui produisent l'impression ondulatoire. Comme dans la pratique des sérialistes de l'époque, une série est produite dans sa version «droite» et "rétrograde». Dans les ondes symétriques (nommées Ao-VI/o-2), la longueur contient également la série rétrograde (croissante/décroissante), tandis que dans les ondes asymétriques (nommées Bo-VI/o-2) la longueur contient seulement la série ascendante (ou descendante); par exemple: Ao/o symétrique: 17, 27, 43, 70, 113, 70, 43, 27, 17; Bo/o asymétrique: $17,27,43,70,113$.

Toujours à l'instar d'une conception musicale, une série de 12 notes peut se transposer selon ses 12 degrés. Dans le cas des «ondes» pour les vitres «ondulatoires », on obtient la transposition équivalente ( $1{ }^{\text {ère }}, 2^{\text {ème }}$, etc.) en retirant respectivement les premiers, deuxièmes, etc. termes de la forme d'onde fondamentale équivalente. Xenakis obtient ainsi la ière transposition de l'onde $\mathrm{A} \mathrm{I} / 2$, la 4ème transposition de l'onde $\mathrm{AIV} / 2$, etc. Le début de l'onde transposée est ainsi décalé par rapport au début de l'onde fondamentale; mais à la différence de la pratique compositionnelle, ce décalage ne concerne pas toutes les valeurs de la série: l'onde transposée est plus courte, mais les termes restants sont les 
mêmes que dans la série fondamentale (ex. Bo/o: 17, 27, 43, 70, $113 \rightarrow$ BII/o: 43, 70, 113).

Par souci de formalisation, Xenakis note dans la fraction qui définit le nom de chaque onde (Ao/o, AI/o, Bo/o, BII/o, Ao/1, AIII/1, Bo/1, BII/1, Ao/2, AI/2, $\mathrm{AIV} / 2, \mathrm{AVI} / 2, \mathrm{Bo} / 2, \mathrm{BI} / 2, \mathrm{BIV} / 2, \mathrm{BVI} / 2$ ) ses caractéristiques. Ainsi le numérateur de la fraction définit la classe de l'onde [fondamentale (o) ou Nième transposition (I, II, III, IV etc.], tandis que le dominateur $(0,1,2)$ définit la série du Modulor qui a été utilisée [o pour la rouge, 1 pour la bleue, 2 pour leur combinaison].

En bas de ce plan à gauche, Xenakis donne un exemple de mélange $(C)$ de différents segments d'ondes. Il est intéressant de souligner qu'il n'utilise pas en entier les ondes qu'il vient d'exposer: "juxtaposition des fragments des $\mathrm{An} / \mathrm{m}$ et $B n / m »$ répète son texte dans le brevet. Dans cet exemple (C), il prescrit une saccade entre deux fragments, si le mouvement ascendant ou descendant d'une onde s'oppose au mouvement inverse de l'onde suivante; ou bien on y voit une continuation, si le mouvement ascendant ou descendant d'une onde suit le mouvement de l'onde voisine. Xenakis propose aussi un mélange d'ondes (D) en bas à droite qu'il appelle «oppositions de structures». On y aperçoit de grandes concentrations de petites distances $(\gamma)$ - ce qui est l'équivalent d'une grande densité de membrures de béton - délimitées par une structure $(\delta)$, dotée d'une faible densité en membrures de béton. On peut s'interroger sur la manière dont ces structures denses sont produites; Xenakis dans ce tableau ne mentionne nulle part selon quel principe il modifie la densité. Et cela reste un mystère, car dans son texte de 1984, il ne dévoile pas non plus le principe selon lequel il a opposé les parties denses des membrures en béton à des raréfiées sur les façades extérieures du couvent.

\subsubsection{Les différentes versions en pans de verre «ondulatoires » des façades extérieures}

Le tableau des « ondes», que nous venons de présenter [fig.9], date de juin 1955 (23-06-1955), mais son apparition vient sans doute simplement "officialiser» le travail de Xenakis. Les premiers plans, qui montrent un changement dans le traitement des façades extérieures, datent de février 1955. Nous rappelons que les façades extérieures jusqu’à la fin 1954 (19-11-1954) ont été vitrées avec des pans «combinatoires». Comme nous l'avons vu, Le Corbusier, qui était de retour de Chandigarh au début du printemps 1955 (fin février), apporte avec lui un croquis de Pierre Jeanneret sur la façon dont les maçons indiens économisent les chutes de verre.

Le plan $\mathrm{N}^{\circ} 2553$ (24-02-1955) [fig.10] présente la façade ouest avec quatre étages; sa version est barrée et accompagnée de la date et du nom de Xenakis. Sur les troisième et quatrième étages, nous distinguons neuf cases qui accueillent des «ondes» de verre. En effet, chacune de ces cases mesure autant que deux cellules du cinquième ou du sixième étage. Une autre pratique d'architecte se manifeste ici: Xenakis prend comme référence les lignes des éléments déjà dessinés (ici les cellules) pour faciliter la distribution des vitres. D’ailleurs, ces cases sont les mêmes que celles qui ont été remplies par des pans 


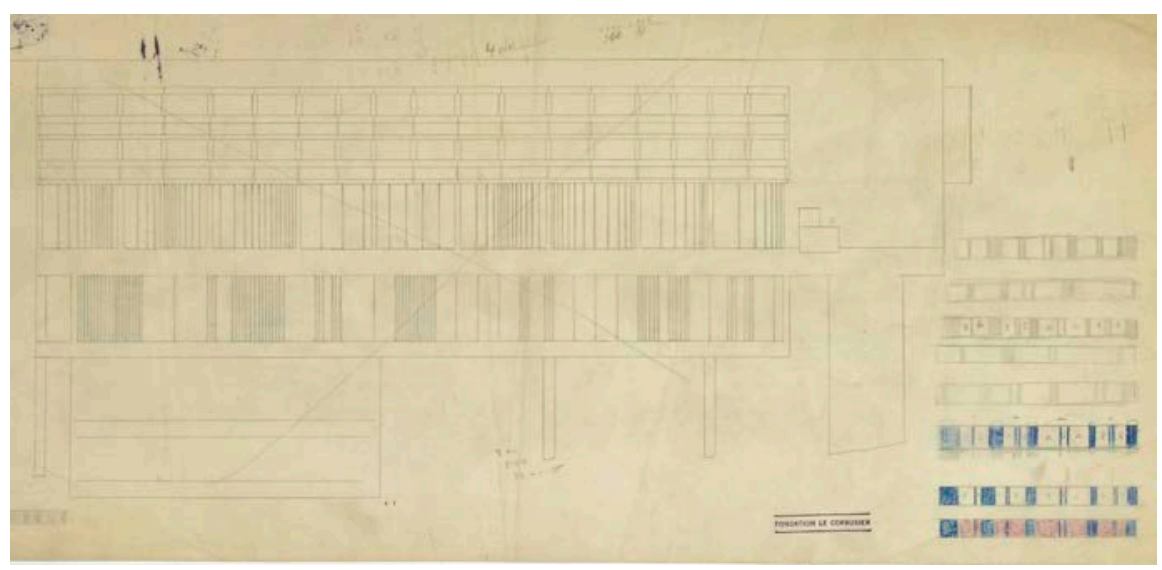

Figure 10. Étude par Xenakis pour les pans de verre de la façade ouest $\mathrm{N}^{\circ} 2553,24-02-1955$, (C) F.L.C. / Adagp, Paris, 2017.

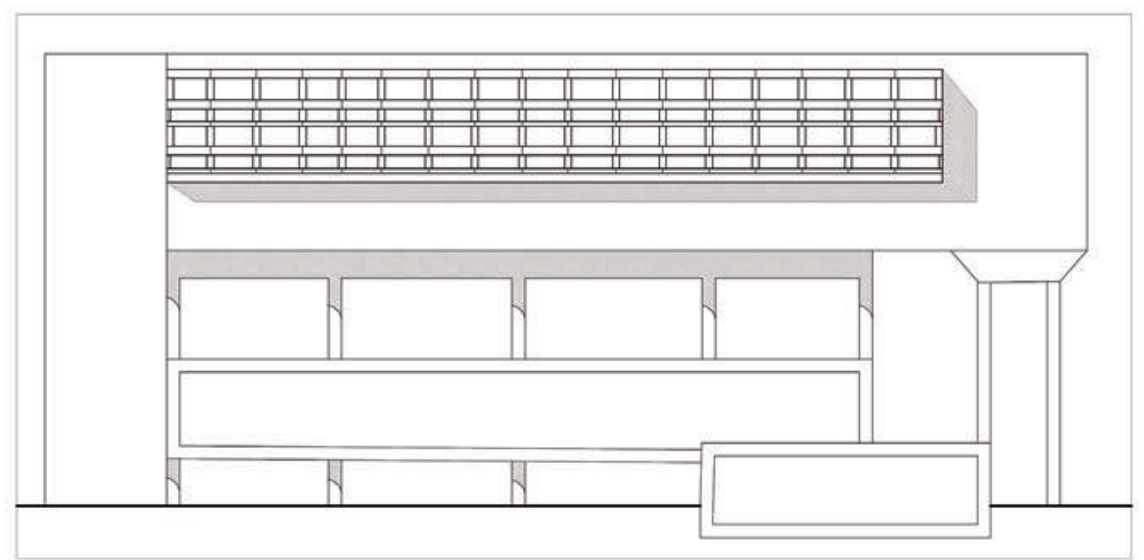

Figure 11. Élévation de la façade ouest par Xenakis et Le Corbusier, 11/12-05-1954. Copie établie par Elisavet Kiourtsoglou d'après le plan original $\mathrm{N}^{\circ} 1333$, FLC.

«combinatoires» lors des premiers essais de Xenakis pour étudier le vitrage des façades extérieures. Nous renvoyons le lecteur au plan $\mathrm{N}^{\circ} 1333(11-12 / 5 / 1954)$ [fig.11] qui représente une version antérieure de la façade ouest (printemp. 1954), où les trois étages inférieurs portent les cases des vitres, qui se séparent en accord avec la division des cellules des deux derniers étages et les cinq poteaux de la structure portante. Xenakis cherche à emboîter à l'intérieur de ces cases une certaine forme de vitres, qui à ce moment, n'est pas encore arrêtée; plus tard il va y faire entrer des pans de verre "combinatoires".

De retour au plan $\mathrm{N}^{\circ} 2553$ [fig.10], en bas à droite, dans des croquis à main levée, Xenakis révèle la clé de l'opération de mélange des ondes à laquelle il procède. Ainsi, il juxtapose des parties denses (au crayon bleu) aux parties raréfiées (au crayon rouge); ces dernières prennent quatre longueurs distinctes ( $\mathrm{a}, \mathrm{b}$, c, d). Dans ce croquis, Xenakis ne montre pas les éléments qui constituent les 
ondes, mais seulement l'opposition de densité. Ce qui est l'objet de son attention se situe à un niveau plus élevé; il s'agit de la longueur globale (ou la durée si l'on peut dire) à l'intérieur de laquelle va s'étaler une grande densité de membrures de béton. Sur le plan principal maintenant, le rythme élémentaire, qui se produit par l'opposition des structures denses et raréfiées, a toujours lieu à l'intérieur des neuf cases (voir troisième étage). Ce choix produit un effet ondulatoire très répétitif, qui aurait dû être une des principales raisons de l'échec de cette proposition.

Le plan $\mathrm{N}^{\circ} 2557$ (24-02-55) [fig.12] porte également la même date que le plan $\mathrm{N}^{\circ} 2553$ et présente une autre tentative d'organiser la façade ouest du couvent. Il s'avère que cette date correspond au moment où Xenakis abandonne le plan $\mathrm{N}^{\circ} 2553$ pour le plan $\mathrm{N}^{\circ} 2557$. En effet, il s'agit d'une pratique d'organisation assez courante dans l'atelier Le Corbusier de signaler le moment de l'annulation/ modification d'un plan pour que les autres collaborateurs ne le prennent pas en considération dans leur étude. C'est pour cette raison que nous pensons que le plan $\mathrm{N}^{\circ} 2553$ présente la première proposition faite par Xenakis pour la façade ouest (réalisée probablement au début du mois de février et assurément avant le 24-02-1955) et non la deuxième comme le suggère Barthel-Calvet (2009, p. 19). Cette hypothèse se confirme encore dans la mesure où, dans le plan récapitulatif $\mathrm{N}^{\circ} 1340$ (15-5-1955), la façade ouest dessinée coïncide avec la version du plan $\mathrm{N}^{\circ} 2557$; cela veut dire que c'est la version du $\mathrm{N}^{\circ} 2557$ qui a été gardée après le 2202-1955. D’ailleurs, dans le livre de Le Corbusier Modulor 2, achevé le 12-05-1955, le projet de pans de verre ondulatoires de la Tourette est présenté dans la version du plan $\mathrm{N}^{\circ} 2557$. Enfin, le plan $\mathrm{N}^{\circ} 2559$, qui porte la même date (24-02-1955) et qui est un tirage du $\mathrm{N}^{\circ} 2557$, montre la même version de la façade ouest avec

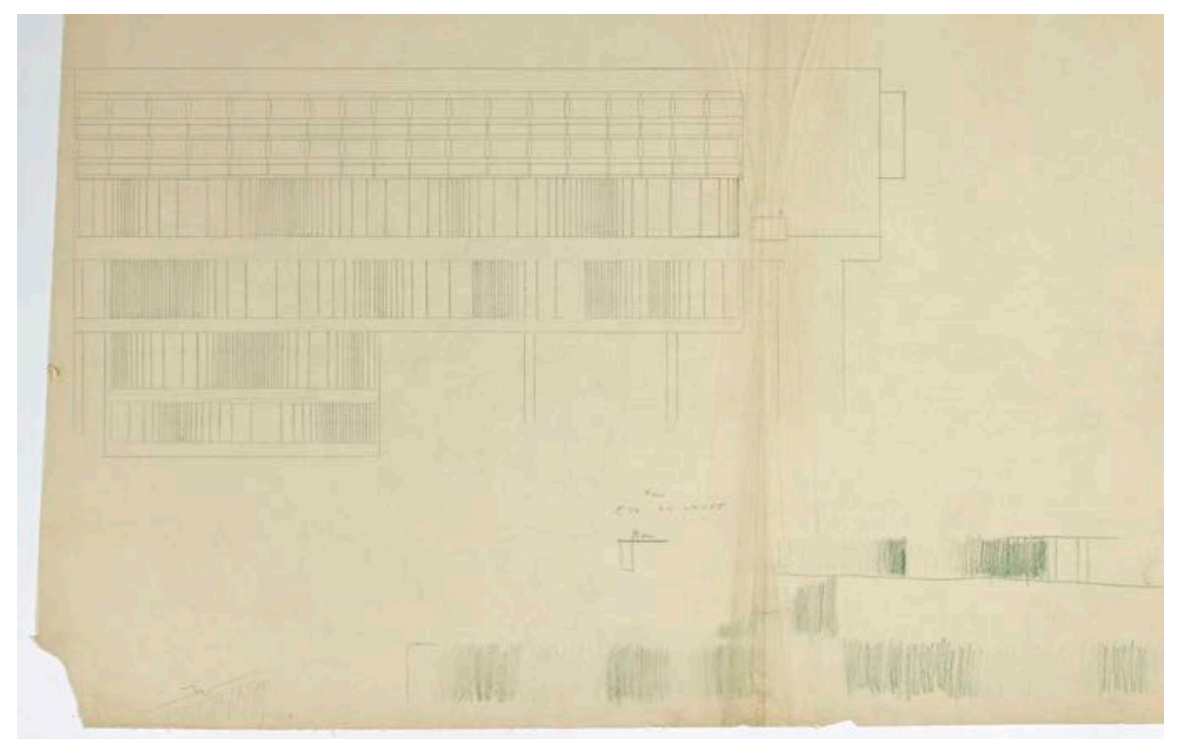

Figure 12. Étude par Xenakis pour les pans de verre de la façade ouest, $\mathrm{N}^{\circ} 2557,24-02-55$, (C) F.L.C. / Adagp, Paris, 2017. 
des annotations au crayon marron pour indiquer les pans de verre qui servent d'aération; ce qui vient renforcer notre hypothèse, puisqu'il est plus probable que la façade ait déjà été arrêtée le 24 février afin que Xenakis ait pu y indiquer les pans ouvrants le même jour. Cette version de la façade ouest $\left(\mathrm{N}^{\circ}{ }_{2557}\right)$ a perduré jusqu'à novembre 1956, lorsque Xenakis a produit la version qui a été finalement construite. Pendant longtemps, dans l'atelier, la version de $\mathrm{N}^{\circ} 2557$ a été considérée comme définitive, c'est d'ailleurs peut-être pour cette raison que l'on trouve un tirage de ce plan en encre de chine dans la collection privée de la famille Xenakis [fig.13], utilisé également dans la deuxième édition du livre de Xenakis Musique/Architecture en 1976 [fig.14]. Mais comment Xenakis a-t-il laissé derrière lui le plan $\mathrm{N}^{\circ} 2253$ [fig.10], où il envisageait des permutations des parties denses et raréfiées à l'intérieur des cases en correspondance avec les cellules juste en-dessus, pour passer au plan $\mathrm{N}^{\circ} 2557$ [fig.12] qui fait preuve d'une manipulation «ondulatoire» beaucoup plus fluide?

Le plan $\mathrm{N}^{\circ} 2554$ (sans date) [fig.15], comporte une étude des degrés de densités de la façade ouest. Sur ce plan, qui accompagne le texte de Xenakis (figure 13) dans les Garland Architectural Archives, l'architecte juxtapose des parties denses à des parties raréfiées en y modifiant le degré de "noirceur» du trait de sa plume. On a l'impression que Xenakis commence à envisager une étude rythmique élémentaire, fondée sur l'alternance de l'intensité des parties sombres (denses) et claires (raréfiées). Dans la musique, ceci renvoie aux nuances, l'action de varier l'intensité dans une phrase musicale en incitant les instrumentistes à jouer plus ou moins fort (forte, fortissimo, piano, pianissi$m o$, etc.). Leurs changements progressifs ou soudains (crescendo, decrescendo, sforzando, etc.) sont utilisés comme accents d'une phrase musicale et très souvent, dans la musique du $\mathrm{XX}^{\mathrm{e}}$ siècle, servent aussi d'élément rythmique au sein d'une composition. Peut-être n'est-ce pas un hasard si Fernand Gardien - un autre ancien de l'atelier Le Corbusier - se rappelle que Xenakis «battait systématiquement la mesure en chantonnant ${ }^{15}$ » pendant l'organisation des façades en pans de verre "ondulatoires».

C’est seulement le plan $\mathrm{N}^{\circ} 2560$ (sans date) [fig.16] qui montre concrètement ce travail de dynamiques (forte, fortissimo, piano, pianissimo, etc.) au-dessus de chaque étage de la façade ouest. Malheureusement on ne peut pas l'examiner plus en détail puisqu'il s'est perdu au fil du temps ${ }^{16}$. À partir de sa seule reproduction, nous pouvons constater effectivement les crescendos et les decrescendos au-dessus de chaque étage de la façade ouest pour passer d'une densité ( $f=f o r t e, f f=f$ ortissimo, etc.) à l'autre, en utilisant les soufflets de l'écriture musicale. L'analogie se construit ici entre la densité architecturale et l'intensité musicale; une forte intensité musicale, $\{f\}$ et $\{f f\}$, vaut pour une grande densité en membrures de béton (ou par équivalence une grande concentration de petites distances de verre: 13, 17, 20, $27 \mathrm{~cm}$ ). Un autre élément significatif

15 Ferro et al. 1987, p. 91.

16 Le plan $\mathrm{N}^{\circ} 2560$, qui accompagnait le texte de Xenakis (figure 12) dans l'édition des Garland Architectural Archives (1984), ne peut pas être localisé dans les archives de la fondation Le Corbusier à l'heure actuelle. Selon les responsables de la fondation, il a été probablement perdu pendant la prise de vue pour le tome. 


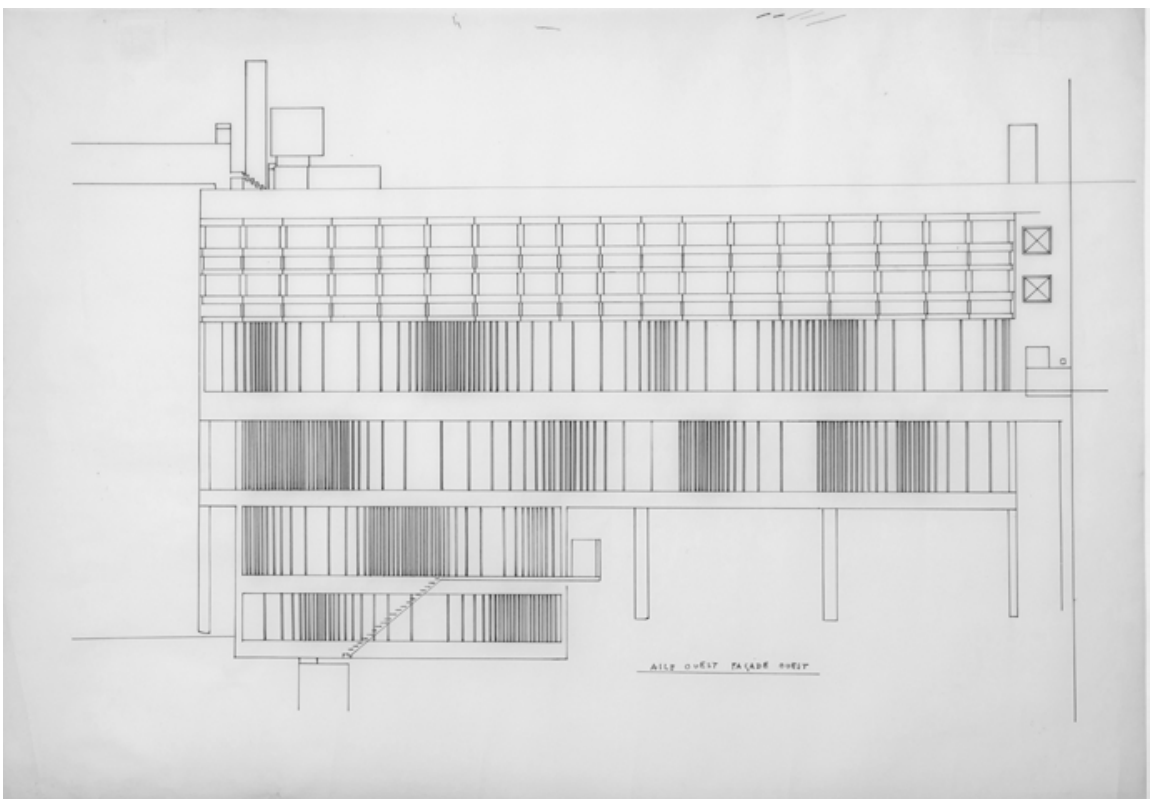

Figure 13. La façade ouest du couvent, Archives de la famille Xenakis. Avec l'aimable autorisation de la famille Xenakis.

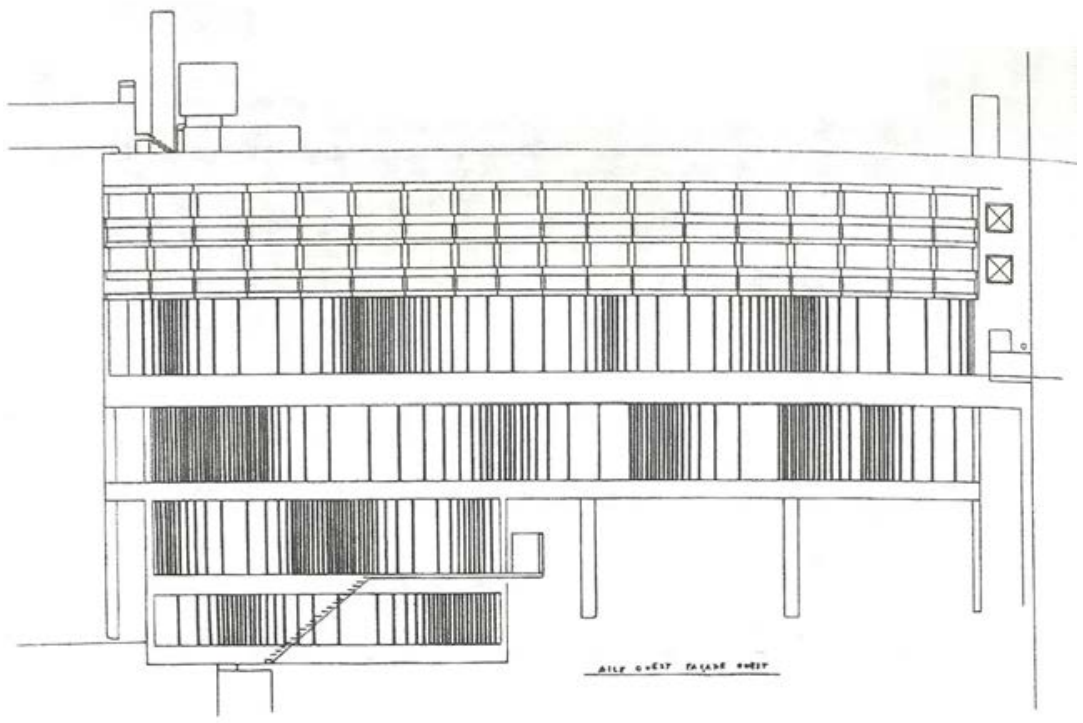

Couvent de Sainte-Marie-de-la-Tourette à Eveux sur l'Arbresle, 1955. Pans de verre ondulatoires, façade ouest. Voir p. 150.

Figure 14. Extrait de l'ouvrage Musique/Architecture, deuxième édition, 1976, p. 166 de Xenakis où il figure la façade ouest du couvent (C) Casterman. Avec l'aimable autorisation des auteurs et des Éditions Casterman. 


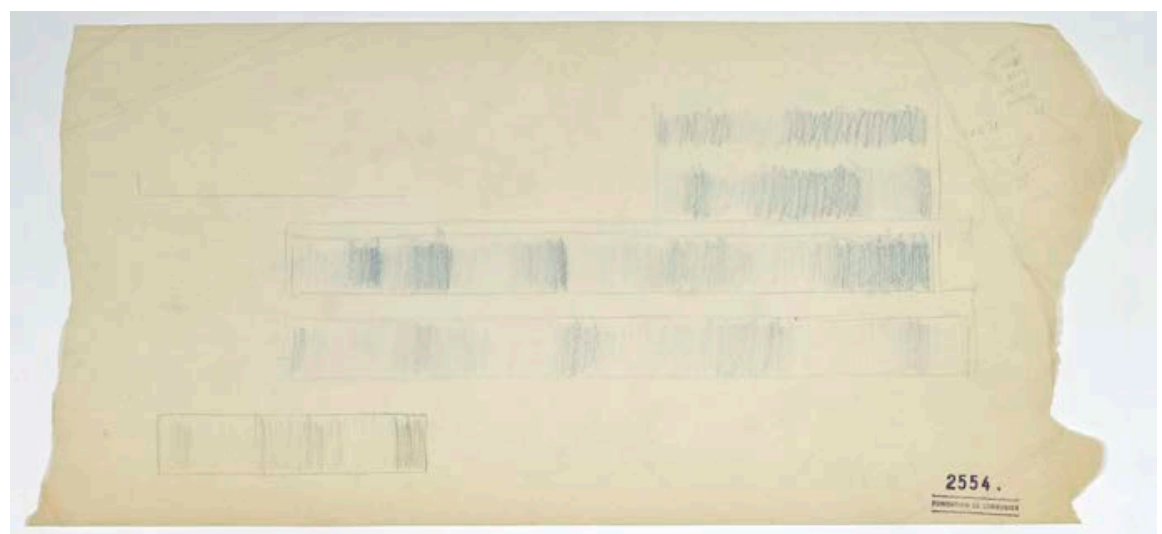

Figure 15. Étude de densité par Xenakis pour la façade ouest, $\mathrm{N}^{\circ} 2554$, sans date, ( F.L.C. / Adagp, Paris, 2017.

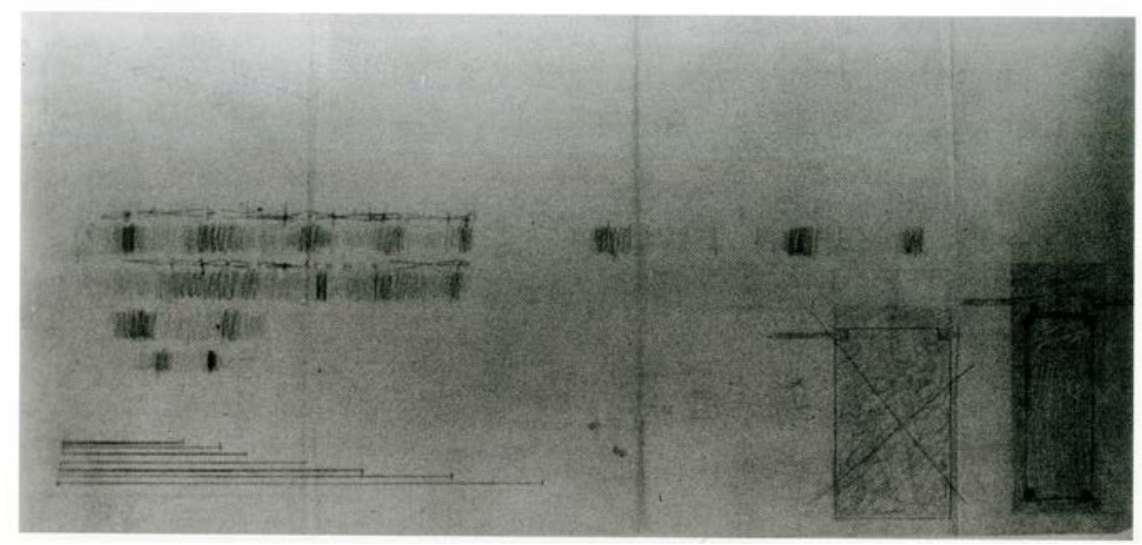

Figure 16. Travail de dynamiques (forte, fortissimo) au-dessus de chaque étage de la façade ouest par Xenakis, $\mathrm{N}^{\circ} 2560$, sans date (C) F.L.C. / Adagp, Paris, 2017.

de l'analogie qui est en train de se nouer ici, se trouve en bas à gauche de ce plan. Nous y voyons un nombre de lignes droites de longueur graduellement variable, empilées à la verticale. Malheureusement, il n’y a aucune échelle et aucun chiffre qui aurait pu nous permettre d'indiquer ces valeurs. En revanche, nous pouvons retrouver certaines de ces longueurs - en tant que valeurs absolues - sur le croquis de la façade en haut de ce plan. Là, elles coïncident avec la durée (= longueur) pendant laquelle les différents changements d'intensité (= densité) se déploient. Cela produit un compartimentage de la façade en segments à l'intérieur desquels se produit le passage des zones en faible densité à celles dotées d'une grande densité. Or, la longueur de l'«ondulation» des densités sur les façades correspond aux durées du changement d'intensité dans 
la musique; ainsi toutes les densités sur la façade sont dotées d'une longueur (durée) spécifique, comme l'indique d'ailleurs Xenakis dans le texte de $1984^{17}$.

D'ailleurs, au début de la partition de Metastaseis $^{B}$ Xenakis fait le rapprochement entre la conception des façades du couvent et la composition de cette pièce, en soulignant ce travail commun sur les durées (longueurs) de dynamiques:

Des structures d'intervalles, de durées de dynamiques et de timbres sont combinées en faisant appel aux progressions géométriques, en particulier à la section d'or, conceptions analogues à celles que l'auteur appliquait en dessinant les façades du Couvent de la Tourette près de Lyon. (Xenakis 1963 , nous soulignons).

Entre mai 1955 et février 1956, la production de plans sur les façades stagne. Ceci est sans doute à mettre en rapport avec l'avancement du chantier et les divers problèmes financiers qui sont apparus ${ }^{18}$. En février 1956, le Couvent a toujours quatre étages au-dessus des cellules, mais sa hauteur va changer vers

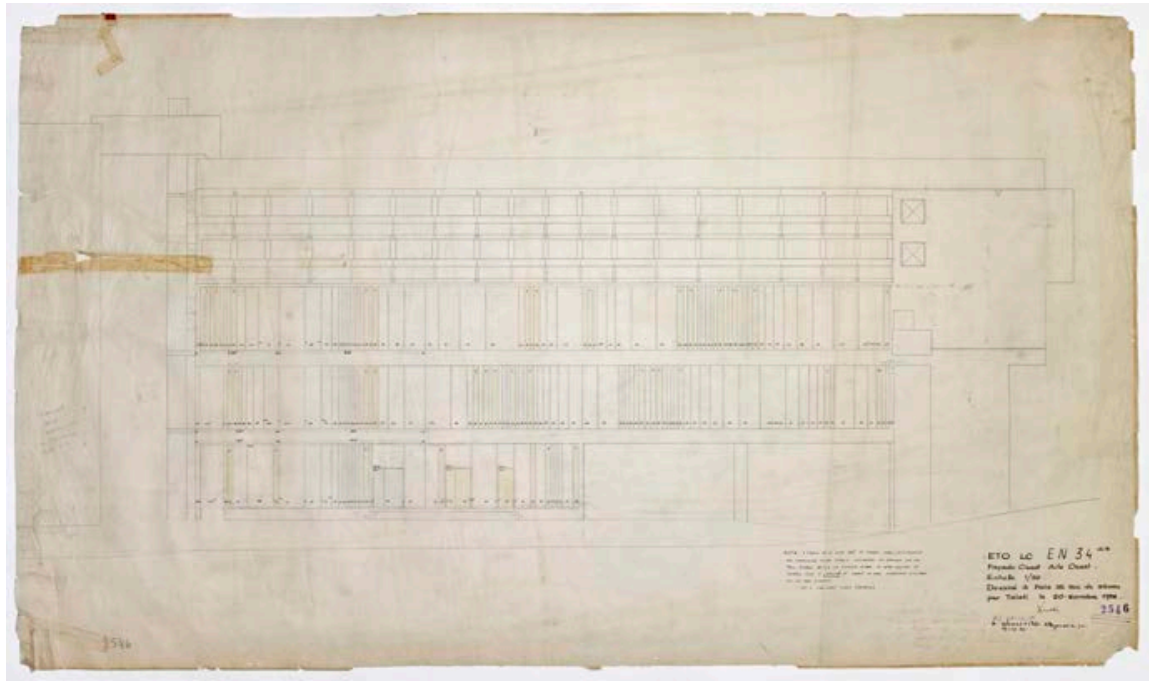

Figure 17. La version définitive de la façade ouest $\mathrm{N}^{\circ} 2546$, signée par Xenakis, 20-11-1956, (c) F.L.C. / Adagp, Paris, 2017.

la mi-avril, après la décision de Le Corbusier de l'abaisser d'un étage en vue d'importantes économies.

Dans les plans définitifs du couvent, datés du 20-11-1956, la façade sud $\left(\mathrm{N}^{\circ} 2543\right)$ et la façade est $\left(\mathrm{N}^{\circ} 2545\right)$ n’ont pas été retravaillées, étant donné que leur forme n’a pas été affectée par la modification de la hauteur des pilotis.

17 Xenakis 2006, p. 116, nous soulignons : «Donc la solution est de juxtaposer des parties denses (beaucoup de montants en béton armé) à des raréfiées. Ici, naturellement, il faut définir les degrés de densité et leurs longueurs (durées)."

18 L'avancement du chantier pendant cette période est détaillé dans Ferro et al. 1987, p. 35-37. 
Seule la façade ouest, qui a perdu un étage, a dû être revue et sa version finalement construite est celle du plan $\mathrm{N}^{\circ} 2546$ (20-11-1956), [fig.17].

Dans un premier temps, il nous est difficile de saisir les règles qui régissent la répartition des verres «ondulatoires» sur le plan définitif $\mathrm{N}^{\circ} 2546$; de surcroît, les formes d'onde décrites dans le tableau de brevet [fig.9] n'y apparaissent pas entièrement. Devant une organisation si peu régulière, les chercheurs se posent très naturellement la question d'une distribution intuitive ${ }^{19}$ ou "stochastique». D'ailleurs Xenakis, comme on l'a vu, avait déjà exclu l'utilisation des probabilités pour la distribution des dix longueurs de verres sur la façade.

Pourtant, Barthel-Calvet avance l'hypothèse de l'emploi des probabilités non pour la distribution générale des distances, mais pour l'apparition de telle ou telle densité au-dessous des 49 cellules. Malgré son intérêt et de l'aveu même de la chercheuse, cette hypothèse reste inadéquate pour expliquer la distribution des pans de verre (Barthel-Calvet 2009, p. 20-21). Les valeurs de densités attendues, issues d'une telle distribution probabiliste, s'éloignent des valeurs observées sur la façade, mais pas de beaucoup et surtout pas partout. Comme le souligne l'auteur, ces écarts entre les valeurs attendues et observées pourraient rappeler une pratique très courante chez Xenakis; dans son travail de compositeur, Xenakis n'hésite pas à s'éloigner de l'application des lois théoriques strictes au profit de la plasticité de l'ouvre dans son ensemble. Ainsi, l'architecte a peut-être conçu une distribution probabiliste des densités de verre, dont il a remanié les calculs au profit de la plasticité globale de la façade. Pourtant, aucun élément des archives ne vient confirmer son hypothèse.

\subsection{Une hypothèse pour les pans de verre "ondulatoires"}

Au premier abord, les formes d'onde - dessinées dans le tableau $\mathrm{N}^{\circ} 3060$ [fig.9] qui accompagne le brevet - semblent ne pas être appliquées fidèlement sur le plan $\mathrm{N}^{\circ} 2546$ [fig.17]. Il y a des ondes auxquelles il manque certaines distances pour se compléter et d'autres qui ne respectent pas l'ordre ascendant ou descendant prescrit dans le brevet. Il existe même des distances solitaires qui semblent n'appartenir à aucune forme d'onde connue. S'agit-il ici encore d'un exemple où Xenakis établit un système théorique pour le briser ensuite, pendant sa mise en place, en faveur de la plasticité de l'œuvre?

Le plan $\mathrm{N}^{\circ} 31598$ (sans date) [fig.18] nous aide à signaler un élément qui est passé inaperçu auprès des chercheurs. La disposition des pans de verre dans ce plan est exactement la même que dans la version finale du plan $\mathrm{N}^{\circ} 2546$; cependant, les trois étages de la façade ouest, dessinés au crayon noir, sont divisés en segments, par un léger trait de plume bleu. Nous les transcrivons ici:

3ème étage: $4.735,8.36,13.16,5.04,8.37$

19 Sterken 2004, p. 129, nous soulignons: «Une fois effectuées ces opérations sur le matériau de base, le travail de composition proprement dit consiste en la juxtaposition, la superposition ou la confrontation, dans la façade, des séquences ainsi obtenues, suivant l'aspect global de la composition déterminé avant. Curieusement, celui-ci semble avoir été déterminé de façon plus ou moins intuitive, à main libre-cela pourrait expliquer pourquoi on n'a pas pu déterminer des rapports quantifiables dans la composition des pans de verre à l'échelle de la façade». 


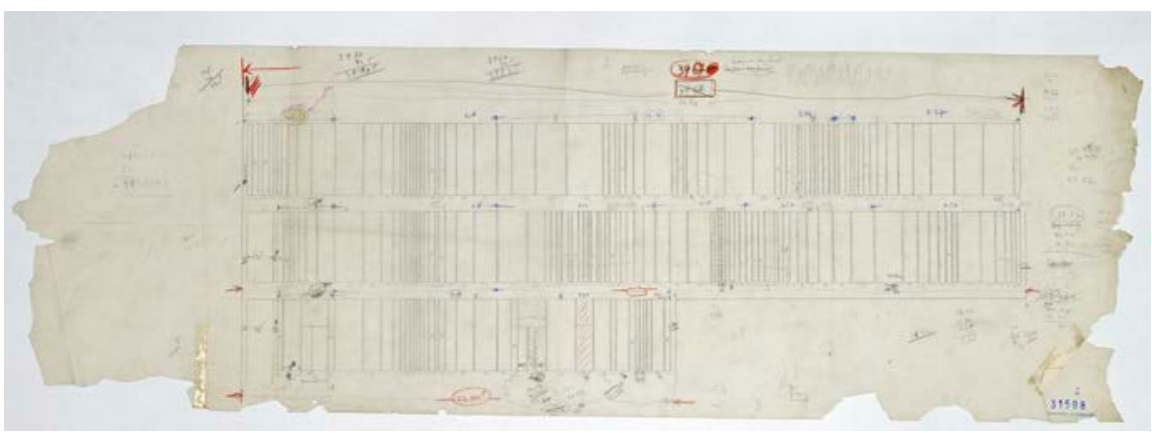

Figure 18. La division de la façade ouest en segments par Xenakis, $\mathrm{N}^{\circ} 31598$, sans date, () F.L.C. / Adagp, Paris, 2017.

2ème étage: $2.95,8.36,8.14,4.33,6.57,7.50$

1ère étage: $2.95,8.36,8.71$

Force est de constater que ces divisions «oscillent» autour des valeurs de la série rouge de Modulor (2,96, 4,79, 7,74, 12,53). C'est à l'intérieur de ces segments, que nous arrivons à indiquer une ou plusieurs ondes, selon la nomenclature du tableau de brevet. C'est-à-dire que Xenakis abandonne les «cases» en accord avec les cellules de l'étage au-dessus pour produire un compartimentage qui suit d'une certaine façon le Modulor; et cela lui fait éviter l'aspect très répétitif qui se produisait dans ses premiers essais. Mais il faut également noter qu'à l'intérieur de ces segments, nous n'allons pas trouver des ondes entières ou leur forme «officielle» présentée dans le tableau de brevet $\mathrm{N}^{\circ} 6030$. Xenakis procède à une transformation des ondes, que nous croyons avoir décryptée ici.

Nous allons procéder à une analyse de segments sur le plan $\mathrm{N}^{\circ} 31598$ pour y faire apparaître les formes d'ondes et leurs "transformations». Ci-dessous, les chiffres soulignés correspondent aux éléments communs entre a) les formes d'onde initialement décrites dans le tableau $\mathrm{N}^{\circ} 6030$ et b) celles constatées sur la façade finale des plans $\mathrm{N}^{\circ} 2546$ et $\mathrm{N}^{\circ} 31598$. En outre, les chiffres soulignés du deuxième rang sont le produit d'une «transformation » des éléments non apparus de l'onde initiale (premier rang). Par exemple:

\section{зème étage (de gauche à droite)}

- Segment $4.735\left(4.51+0.225^{20}\right)$ :

Théorique $^{21}$ : 0.170 .270 .430 .701 .130 .700 .430 .27 (onde $\mathrm{A}^{\%} \%$ ) + 0,06* 8 (béton) $=4,58 \mathrm{~m}$

Observé: $\underline{0.20} 0.270 .170 .270 .170 .130 .170 .270 .430 .701 .13+0,06^{\star} 11=4,57 \mathrm{~m}$

Longueur de densité observée: $0.200 .270 .170 .270 .170 .13+0,06^{\star} 6=1,57 \mathrm{~m}$

20 Il s'agit de la longueur de colonne tout à gauche de la façade, que Xenakis prend en compte pour ce segment.

${ }^{21}$ Selon la nomenclature du tableau $\mathrm{N}^{\circ} 6030$, qui accompagne le brevet des pans de verre «ondulatoires». 
Longueur «transformée» de l'onde initiale $\mathrm{A} \%$ : $0.70,0.43,0,27+$ $0,06^{\star} 3=1,58 \mathrm{~m}$

Nous montrons ici qu'effectivement la partie dense, formée par les six éléments soulignés $(0.20,0.27,0.17,0.27,0.17,0.13)$, est produite par un «morcellement» des éléments non apparus de la série initiale $\mathrm{A} \%$ (0.70, 0.43, 0,27). Ici, il faut ajouter les membrures de béton (0,06 entre chaque élément) : $3^{\star} 0,06=0,18$ pour le premier rang, $6^{\star} 0,06=0,36$ pour le deuxième, à la suite de l'insertion de longueurs de verre supplémentaires. Ainsi, la partie dense mesure $1,57 \mathrm{~m}$, tandis
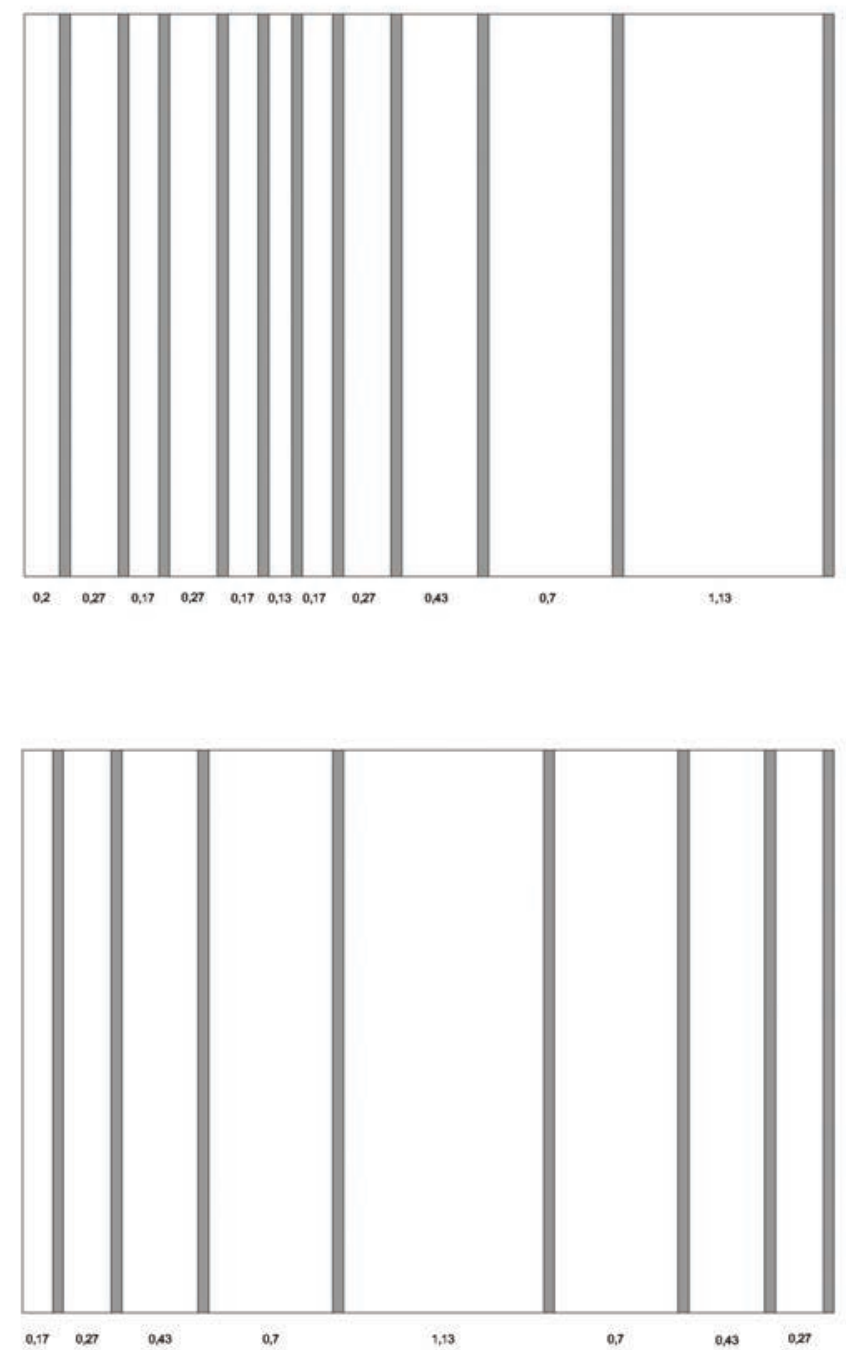

Figure 19. La transformation d'une onde. Graphique établi par Elisavet Kiourtsoglou. 
que les éléments non apparus de la série initiale A\% mesureraient 1,58m. Cela signifie que la partie dense est formée à l'intérieur d'une "onde » avec la division des grands pans de verre en longueurs plus petites et en y ajoutant la longueur des membrures de béton équivalente [fig.19]. Nous proposons de déchiffrer ainsi toute la façade ouest; nous divisons les segments indiqués en plusieurs tronçons, selon le nombre de formes d'ondes que nous arrivons à y distinguer.

\section{3ème étage (de gauche à droite)}

- segment 4.735(4.51+0.225):

Théorique: $0.17 \quad 0.27 \quad 0.43 \quad 0.701 .130 .700 .430 .27$ (onde $\mathrm{A}^{\%} \%$ ) + o,o6 8 (béton) $=4,58 \mathrm{~m}$

Observé: $\underline{0.20} 0.270 .170 .270 .170 .130 .170 .270 .430 .701 .13+0,06^{\star} 11=4,57 \mathrm{~m}$

Longueur de densité observée: $0.200 .270 .170 .270 .170 .13+0,06^{\star} 6=1,57 \mathrm{~m}$

Longueur «transformée» de l'onde initiale $\mathrm{A} \%$ : $0.70,0.43,0,27+$ $0,06^{*} 3=1,58 \mathrm{~m}$

- $\quad$ segment $8,36 \mathrm{~m}$

1er tronçon

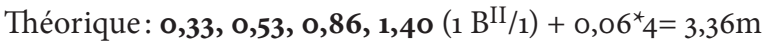

Observé: 1,40, o, 86, o, 53, $0,33^{22}+0,06^{\star} 4=3,36 \mathrm{~m}$

2ème tronçon

Théorique: $0,530,700,861,131,40\left(1 \mathrm{~B}^{\mathrm{VI}} / 2\right)+0,06^{*} 5=4,92 \mathrm{~m}$

Observé: $\underline{0,17} 0,170,130,130,130,130,170,270,130,270,530,861,13+0,06^{\star} 13=$ $5 \mathrm{~m}$

Longueur de densité observée: 0.17 0,17 0,13 0,13 0,13 0,13 0,17 0,27 0,13 0,27 + $10^{*} \mathrm{O}, 06=2,30 \mathrm{~m}$

Longueur «transformée» de l'onde initiale: $1,400,70+0,06^{\star} 2=2,22 \mathrm{~m}$

- segment $13,16 \mathrm{~m}$

1er tronçon

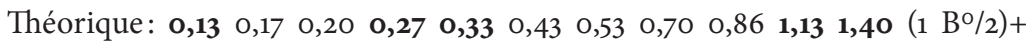
$0,06^{*} 11=6,81 \mathrm{~m}$

Observé: 0,70 1,13 1,40 $\underline{1,83}^{23}$ 0,27 $\underline{0,27} \mathbf{0 , 1 3} \underline{0,27} \mathbf{0 , 3 3}+0,06^{\star} 9=6,87 \mathrm{~m}$

Longueur de densité observée: $1,830,270,27+0,06^{*} 3=2,55 \mathrm{~m}$

Longueur «transformée» de l'onde initiale: $0,170,200,430,530,86+5^{\star} 0,06=$ $2,49 \mathrm{~m}$

2ème tronçon

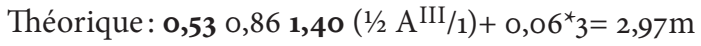

Observé: $0,531,40 \underline{0,20} 0,270,13+0,06^{*} 5=2,83 \mathrm{~m}$

Longueur de densité observée: 0,20 0,27 0,13 + $3^{*} 0,06=0,78$

Longueur «transformée» de l'onde initiale: $0,86+0,06=0,92$

3ème tronçon

22 La forme rétrograde de l'onde BII/1 n'apparaît pas dans le tableau du brevet.

23 La distance 1,83 n'appartient à aucune forme d'onde mais elle fait partie de la série Rouge du Modulor. 
Théorique: $0,33 \mathbf{0 , 5 3} 0,86 \mathbf{1 , 4 0}\left(^{\mathrm{II} / 1}\right)+0,06^{\star} 4=3,36 \mathrm{~m}$

Observé: $0,53 \underline{0,43} \mathbf{0 , 8 6} \mathbf{1 , 4 0}+0,06^{*} 4=3,46 \mathrm{~m}$

Longueur de densité observée: $0,06+0,43=0,49$

Longueur «transformée» de l'onde initiale: $0,06+0,33=0,39 \mathrm{~m}$

- segment 5,04m

Théorique: $0,530,70,861,131,40\left(1 \mathrm{~B}^{\mathrm{VI}} / 2\right)^{24}+0,06^{*} 5=4,92 \mathrm{~m}$

Observé: 1,13 0,27 0,20 0,27 0,20 0,13 0,20 0,17 0,13 0,17 0,27 0,20 0,17 0,27 0,17 $\underline{0,13}+0,06^{*} 16=5,04 \mathrm{~m}$

Longueur de densité observée: 0,27 0,20 0,27 0,20 0,13 0,20 0,17 0,13 0,17 0,27 $0,200,170,270,170,13+0,06^{\star} 15=3,85 \mathrm{~m}$

Longueur «transformée» de l'onde initiale: $0,53+0,70+0,86+1,40+0,06^{*} 4=$ $3,73 \mathrm{~m}$

- segment $8,37 \mathrm{~m}$

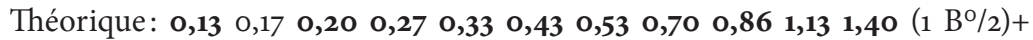
$0,06^{*} 11=6,81 \mathrm{~m}$

Observé: 0,13 0,20 0,27 0,33 0,43 0,53 0,70 o,86 1,13 1,40 (Résidu =1,405) ${ }^{25}$ 0,27

Longueur «transformée» observée: $0,27+0,06=0,33 \mathrm{~m}$

Longueur «transformée» de l'onde initiale: $0,17+0,06=0,23 \mathrm{~m}$

\section{zème étage (de gauche à droite)}

- segment 2,95m

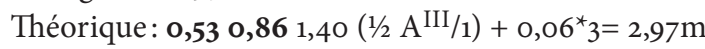

Observé: $\underline{0,27} 0,270,130,200,330,530,86+0,06^{*} 6^{26}=2,95 \mathrm{~m}$

Longueur de densité observée: 0,27 0,27 0,13 0,20 0,33 + 0,06 ${ }^{\star} 4=1,44 \mathrm{~m}$

Longueur «transformée» de l'onde initiale: $1,40+0,06=1,46 \mathrm{~m}$

- segment $8,36 \mathrm{~m}$

1er tronçon

Théorique: $\mathbf{0 , 3 3}, \mathbf{0 , 5 3}, \mathbf{0 , 8 6 , 1 , 4 0}\left(1 \mathrm{~B}^{\mathrm{II}} / 1\right)+0,06^{\star} 4=3,36 \mathrm{~m}$

Observé: 1,40, o,86, $0,53,0,33+0,06^{*} 4=3,36 \mathrm{~m}$

2ème tronçon

Théorique: $0,530,700,861,131,40\left(1 \mathrm{BVI}^{\mathrm{VI}}\right)+0,06^{\star} 5=4,92 \mathrm{~m}$

Observé: $\underline{0,17} 0,170,130,130,130,130,170,270,130,270,530,861,13+0,06^{*} 13=5 \mathrm{~m}$

Longueur de densité observée: 0.17 0,17 0,13 0,13 0,13 0,13 0,17 0,27 0,13 0,27+ $10^{*} 0,06=2,30 \mathrm{~m}$

Longueur «transformée» de l'onde initiale: $1,400,70+0,06^{\star} 2=2,22 \mathrm{~m}$

- segment $8,14 \mathrm{~m}$

1er tronçon

24 Cela correspond à la fois à $1 / 2$ longueur de $\mathrm{AVI} / 2$ et à une longueur entière de $\mathrm{BVI} / 2$.

25 On voit ici apparaître une longueur, qui semble n'appartenir à aucune série de deux séries voisines, étant donné que la distance 1,40 est déjà apparue dans l'onde $\mathrm{B}^{\mathrm{VI}} / 2$ et resurgit aussi dans l'onde $\mathrm{B}^{\circ} / 2$. Cette longueur est décrite comme «résidu» sur le plan.

26 Il n'existe pas de membrure de béton au début de l'onde observée, étant donné qu'elle est collée à la colonne. 
Théorique: $0,530,861,40\left(1 / 2 \mathrm{~A}^{\mathrm{III} / 1)}+0,06^{*} 3=2,97 \mathrm{~m}\right.$

Observé: 1,40 0,86 $\underline{0,27} 0,27+0,06^{*} 4=3,04 \mathrm{~m}$

Longueur de densité observée: $0,270,27+0,06^{*} 2=0,66 \mathrm{~m}$

Longueur «transformée» de l'onde initiale: $0,53+0,06=0,59 \mathrm{~m}$

2ème tronçon

Théorique: $0,530,70,861,131,40\left(1 \mathrm{~B}^{\mathrm{VI}} / 2\right)+0,06^{\star} 7=4,92 \mathrm{~m}$

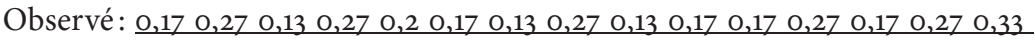

$0,430,53+0,06^{*} 17=5,10 \mathrm{~m}$

Longueur de densité observée: 0,17 0,27 0,13 0,27 0,20 0,17 0,13 0,27 0,13 0,17 $0,170,270,170,270,330,43+0,06^{*} 11=4,51 \mathrm{~m}$

Longueur «transformée» de l'onde initiale: $0,700,861,131,40+0,06^{*} 4=4,3$

- segment $4,33 \mathrm{~m}$

Théorique: $0,53^{27} \mathbf{0 , 7 0} 0,861,131,40\left(1 \mathrm{~B}^{\mathrm{VI}} / 2\right)+0,06^{*} 5=4,92 \mathrm{~m}$

Observé: 0,70 0,86 1,13 0,13 0,130,17 0,20,20,27 + 0,06* ${ }^{*} 9=4,46 \mathrm{~m}$

Longueur de densité observée: $0,130,130,170,20,20,27+0,06^{\star} 6=1,46$

Longueur «transformée» de l'onde initiale : $1,40+0,06=1,46 \mathrm{~m}$

- segment $6,57 \mathrm{~m}$

Théorique: 0,17 0,20 0,27 0,33 0,43 0,53 0,70 0,86 1,13 1,40 $\left(1 \mathrm{~B}^{\mathrm{I}} / 2\right)+0,06^{*} 10=$ $6,62 \mathrm{~m}$

Observé: $0,270,270,27$ 0,20 0,17 0,13 0,17 0,27 0,33 0,43 0,53 0,70 0,86 1,13+ $0,06^{\star} 14=6,57 \mathrm{~m}$

Longueur de densité observée: 0,27 0,27 0,27 0,17 0,13+0,06*5=1,41m

Longueur «transformée» de l'onde initiale: $1,40+0,06=1,46 \mathrm{~m}$

- segment 7,50m

1er tronçon

Théorique: $0,270,430,701,13\left(1 / 2 \mathrm{~A}^{\mathrm{I}} / \mathrm{o}\right)+0,06^{*} 4=2,77 \mathrm{~m}$

Observé : (Résidu 1,39) ${ }^{28}$ o,70 $\underline{0,53} \mathbf{0 , 4 3} \underline{0,33} \mathbf{0 , 2 7} \underline{0,20}+0,06^{*} 6=2,82 \mathrm{~m}$

Longueur de densité observée: $0,530,330,20+0,06^{\star} 3=1,24 \mathrm{~m}$

Longueur «transformée» de l'onde initiale: $1,13+0,06=1,19 \mathrm{~m}$

2ème tronçon

Théorique: 0,17 0,27 0,43 0,70 1,13 (1 $\left.\mathrm{B}^{\circ} \%\right)+0,06^{\star} 5=3 \mathrm{~m}$

Observé: 1,1370 $432717+0,06^{\star} 5=3 \mathrm{~m}$

\section{1ère étage (de gauche à droite)}

- segment 4,735

Théorique: $0,530,861,40\left(1 / 2 \mathrm{~A}^{\mathrm{III} / 1)}+0,06^{*} 3=2,97 \mathrm{~m}\right.$

Observé: $\underline{0,27} 0,751,480,27+0,06^{*} 3=2,95 \mathrm{~m}$

- segment 8,36

1er tronçon

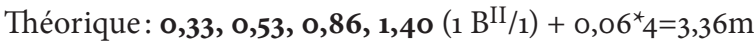

27 Le 0,53 est une distance-pivot, qui participe aux deux séries $\mathrm{B}^{\mathrm{VI}} / 2$ qui se succèdent.

28 La distance 1,39 est décrite comme «résidu» sur le plan $\mathrm{N}^{\circ} 2546$. Elle ne participe pas à la fabrication de l'onde Ao/o. 
Observé: 1,40, o,86, $0,53,0,33+0,06^{*} 4=3,36 \mathrm{~m}$

2ème tronçon

Théorique: $0,530,700,861,131,40\left(1 \mathrm{BVI}^{\mathrm{VI}}\right)+0,06^{*} 5=4,92 \mathrm{~m}$

Observé: $\underline{0,17} 0,170,130,130,130,130,170,270,13\left(1,70+0,04^{*} 2\right)^{29} 1,13+0,06^{*}$ $11=5 \mathrm{~m}$

Longueur de densité observée: 0,17 0,17 0,13 0,13 0,13 0,13 0,17 0,27 0,13 (1,70+ $\left.0,04^{*} 2\right)+0,06^{*} 10=3,81 \mathrm{~m}$

Longueur «transformée » de l'onde initiale: 0,53 o,70 o,86 1,40 + o,o $6^{\star} 4=3,73 \mathrm{~m}$

- segment 8,71

1er tronçon

Théorique: $0,270,430,701,13\left(1 / 2 \mathrm{~A}^{\mathrm{I}} / \mathrm{o}\right)+0,06^{*} 4=2,77 \mathrm{~m}$

Observé: $0,700,27 \underline{\left(0,530,86+0,04^{\star} 2\right)^{30}}+0,06^{\star} 3=2,62 \mathrm{~m}$

Longueur "transformée» observée: $\left(0,530,86+0,04^{*} 2\right)+0,06^{*} 2=1,59 \mathrm{~m}$

Longueur «transformée» de l'onde initiale: $0,431,13+0,06^{\star} 2=1,68 \mathrm{~m}$

2ème tronçon

Théorique: 0,13 0,20 0,33 0,53 0,86 1,40 0,86 0,53 0,33 0,20 ( $\left.1 \mathrm{~A}^{\mathrm{o} / 1}\right)+$ $10^{*} 0,06=5,97 \mathrm{~m}$

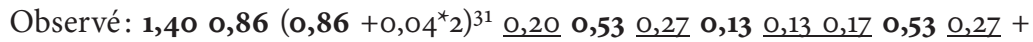
$0,06^{\star} 11=6,09 \mathrm{~m}$

Longueur «transformée» observée: 0,20 0,27 0,13 0,17 0,27 +0,06 ${ }^{\star} 5=1,34 \mathrm{~m}$

Longueur «transformée» de l'onde initiale: $0,200,330,330,20+0,06^{\star} 4=$ $1,30 \mathrm{~m}$

Nous transcrivons ici les formes d'ondes que nous venons de déchiffrer sur la version finale de la façade ouest [fig.2o]. L'existence de distances, appelées "résidus» sur le plan final de la façade [fig.17 \& 18], vient aussi renforcer notre hypothèse. Dans deux cas (premier étage, segment 8,37 , onde $\mathrm{B}^{\circ} / 2$, élément 1,405 et deuxième étage, segment $7,50,1^{\mathrm{er}}$ tronçon, onde $\mathrm{A} / \mathrm{O}$, élément 1,39 ), deux distances, dénommées "résidus", ne participent pas à la fabrication de l'«onde» en question. Ce n'est pas un hasard si ces deux résidus se trouvent à la fin d'une lecture linéaire (de gauche à droite) de chaque étage. En plaçant et en transformant des ondes de gauche à droite, les distances en trop s'accumulent tout à droite de la façade. Ces deux «résidus» ne nuisent en rien à la perception générale, puisqu'elles sont très proches de l'élément 1,40, qui appartient à plusieurs formes d'ondes.

C'est grâce à ces transformations à l'intérieur des "ondes» que nous exposons ici que l'on pourrait également expliquer les différenciations entre le plan d'exécution K1-20-49-001 (28-11-1956) — qui coïncide avec le plan N²546 (2011-1956) - et la construction finale, que nous avons eu l'occasion de mesurer sur place le 19-06-2013. Nous indiquons ces modifications, dues peut-être aux erreurs ou aux nécessités apparues au cours du chantier, en rouge dans ce plan [fig.21]. Le plus souvent il s'agit de distances qui ont été assemblées pour

\footnotetext{
29 La somme de ces distances correspond à la longueur de la porte et l'épaisseur de son châssis.

30 Idem.

31 Idem.
} 


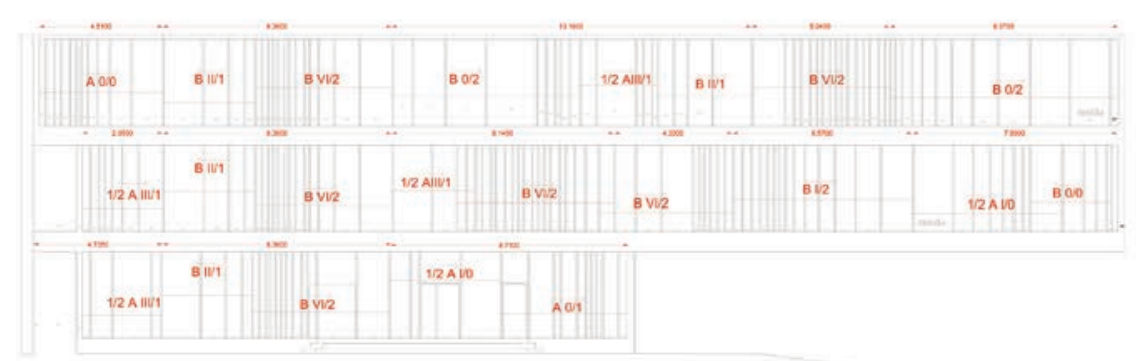

Figure 20. Les formes d'ondes, selon la nomenclature du brevet, déchiffrées sur la version finale de la façade ouest. Plan établi par Elisavet Kiourtsoglou.
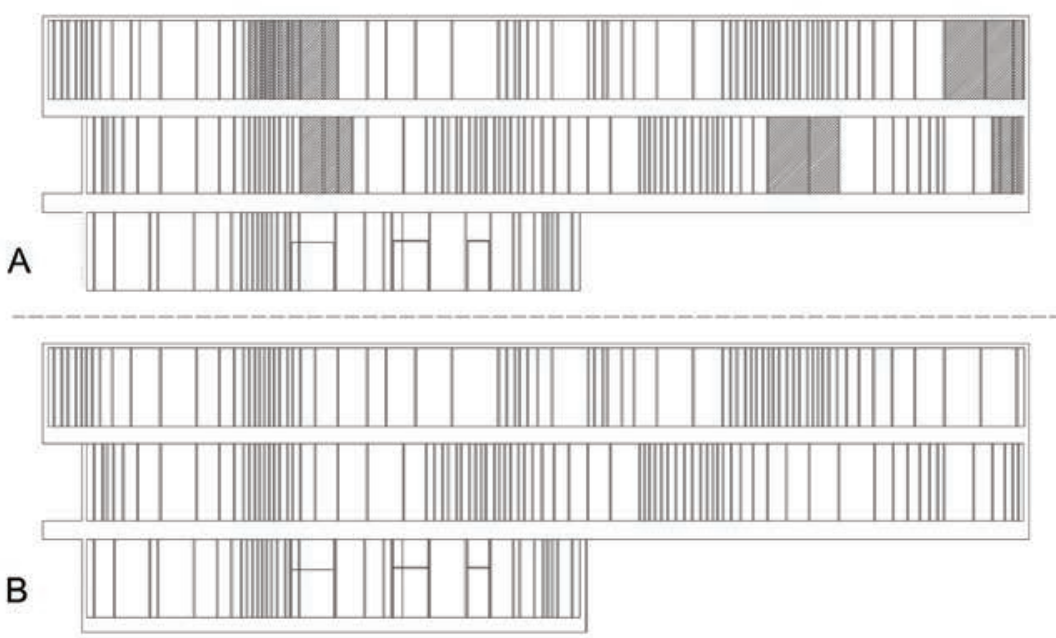

Figure 21. Les différences entre le plan définitif de la façade ouest (B) et la distribution finalement construite (A), après une visite sur place. Plan établi par Elisavet Kiourtsoglou.

produire des pans de verre plus grands (deuxième étage, segment 6,57, éléments $0,70,0,86,1,13$ qui finalement sont transformés en 1,61, 1,14; troisième étage, segment 8,37, éléments 1,40 1,405 0,27 qui sont remplacés par 1,60, 1,07, 0,345). À un autre endroit, deux distances sont morcelées et remplacées par des pans de verre plus petits (deuxième étage, segment $8,36,2$ ème tronçon, éléments $0,53,0,861,13$ remplacés par $0,860,530,530,54$ ). Nous constatons aussi que (troisième étage segment 8,36 , deuxième tronçon) la partie dense est finalement construite par des morceaux de verre encore plus petits que ceux initialement indiqués $(0,20$ 0,17 0,10 0,13 0,10 0,17 0,27 0,13 0,26 au lieu de 0,17 0,13 0,13 0,13 $0,130,170,270,130,27)$. Mais force est de constater que toutes ces modifications se passent à l'intérieur de segments mentionnés auparavant et aux limites de leurs tronçons; c'est-à-dire qu'avec ces modifications on voit une autre transformation possible de la longueur globale des ondes initialement choisies. Et cela 
est possible en utilisant des pans de verre dont la longueur est la somme ou la division de deux ou plusieurs pans de verre.

\subsection{Les analogies entre Metastaseis et les verres "ondulatoires» a) Méthode de sélection des éléments}

Comment Xenakis a-t-il choisi telle ou telle forme d'onde à chaque endroit de la façade? Dans sa musique instrumentale, la question du choix des éléments est résolue, par exemple, dans les années 1960, avec l'utilisation des cribles, qui constituent une méthode de sélection ${ }^{32}$. Dans la musique électroacoustique, à la fin des années 1970, Xenakis choisit l'itinéraire du son d'un haut-parleur à l'autre selon une méthode graphique ${ }^{33}$. Mais en 1956, au moment où les façades du couvent ont été conçues, Xenakis n’a pas eu recours à ces dispositifs. Estce que cela nous permet de conclure qu'il a choisi plus ou moins au hasard, selon son intuition du moment? On devrait écarter cette possibilité, car le hasard pour Xenakis est soit calculé, soit un élément de la nature: dès que la connaissance humaine intervient, c'est-à-dire l'habitude, le hasard s'identifie à l'improvisation et perd son caractère complètement aléatoire ${ }^{34}$. D'ailleurs, on a déjà vu que le compositeur a écarté lui-même la possibilité d'une distribution «stochastique» des verres ondulatoires. Que reste-t-il alors comme option?

Comme nous l'avons signalé, la façade a été divisée en segments, qui «oscillent» autour des termes de la série Rouge du Modulor (2,96, 4,79, 7,74, 12,53).

3ème étage : $4.735,8.36,13.16,5.04,8.37$

2ème étage: $2.95,8.36,8.14,4.33,6.57,7.50$

1er étage: $2.95,8.36,8.71$

Parmi ces segments, certains s'approchent de la somme de deux termes, tirés des séries Bleue et Rouge du Modulor $(9,52+3,66=13,28$ ou $3,66+4,79=8,45)$. Le nota du plan $\mathrm{N}^{\circ} 2546$ se réfère à cette segmentation et est révélateur:

À partir de la cote 4.735 du pignon nord, la distribution des intervalles entre poteaux ondulatoires est identique sur les trois niveaux et sur une distance de 8,36. De cette manière, les poteaux sont à l'aplomb et créent le même mouvement simultané sur les trois niveaux.

En d'autres termes, avec cette segmentation Xenakis contrôle la «durée » des sections, à l'intérieur desquelles des phénomènes ondulatoires se juxtaposent linéairement et verticalement. D'ailleurs dans Metastaseis un compartimentage

32 Dans les mathématiques, les cribles constituent des méthodes de sélection des éléments au sein d'un ensemble. La plus connue, la Crible d'Ératosthène, constitue une méthode de sélection des nombres premiers, par exclusion progressive de tous les nombres divisibles par 2, 3, 5, 7, 11, etc. Xenakis va faire appel aux cribles afin de "construire des symétries aussi complexes qu'on les désire et, inversement, à partir d'une suite donnée d'événements ou d'objets dans l'espace ou dans le temps, de retrouver les symétries qui la constituent.» (Xenakis 1965, p. 75). Le compositeur a utilisé des cribles dans ses compositions à partir des années 1960 (voir Gibson 2011, p. 81-102).

33 Voir Kiourtsoglou, 2017.

34 Voir Delalande 1997, p. 19. 
similaire a été mis en place. Plusieurs chercheurs ont montré qu’une grande partie des Metastaseis se conçoit comme une juxtaposition de sections (différenciées par l'oreille), dont les durées (en mesures) suivent la suite Fibonacci (1, $2,3,5,8,13,21,34,55)^{35}$. Ainsi, par exemple, exemple pour le Violon I, au début des Metastaseis, les glissandi commencent à la première mesure (mesure o) et les clusters vont de 34 jusqu'à la mesure 54 (inclus), donc ils durent 21 mesures. Nous ajoutons des repères rouges sur la partition, reproduite dans le [fig.22].

Rappelons que sur le tableau $\mathrm{N}^{\circ} 6030$ du brevet, les «ondes» se distinguent par rapport à leur longueur globale. En outre, les «transpositions» que Xenakis construit dans ce tableau sont des séries, dont la longueur est plus courte par rapport à celle des ondes fondamentales. De surcroît, toutes les

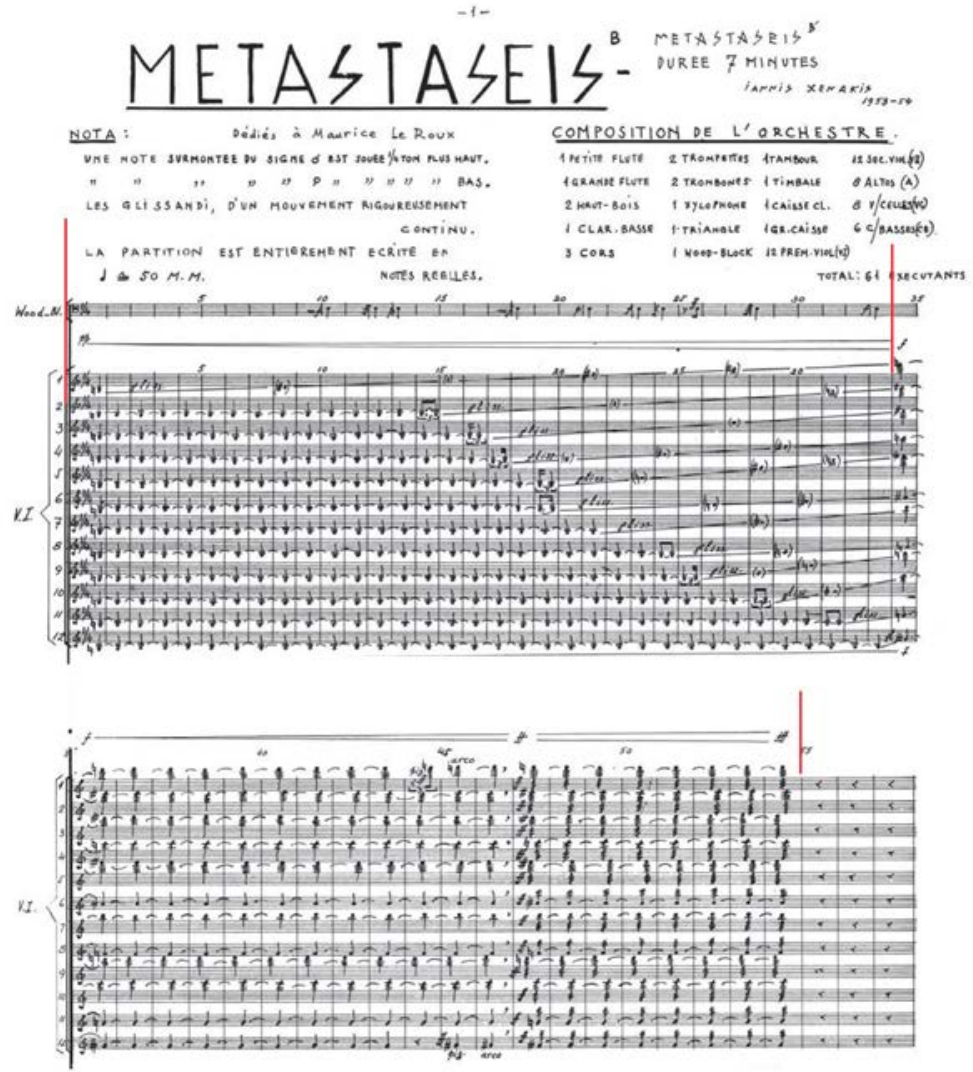

Figure 22 La partition de Metastaseis ${ }^{B}$ et les sections dont les durées (en mesures) suivent la serie Fibonacci $(1,2,3,5,8,13,21,34,55)$. Repères de divisions ajoutés par Elisavet Kiourtsoglou. (c) Copyright 1967 by Boosey \& Hawkes Music Publishers Ltd. Reproduced by permission of Boosey \& Hawkes Music Publishers Ltd.

35 Barthel-Calvet 2000, p. 516. L'auteur renvoie aussi à l'analyse exhaustive de Baltensperger 1996, p. 245-257. 
«transpositions» possibles ne sont pas indiquées; Xenakis choisit celles dont l'élimination des termes produit des diminutions graduelles de la longueur de l'onde fondamentale. Par exemple, dans la famille des ondes créées par la série Rouge du Modulor, il note $\mathrm{A} \%, \mathrm{~A}^{\mathrm{I}} \mathrm{o}, \mathrm{B} \%, \mathrm{~B}^{\mathrm{II}} / \mathrm{o}$; les longueurs des ondes transposées (membrures de béton comprises) diminuent respectivement par rapport à celles des ondes fondamentales (ex. Ao/o :4,58, $\rightarrow \mathrm{AI} / 0: 4,02, \mathrm{Bo} / \mathrm{o}: 3,0 \mathrm{O} \rightarrow$ $\mathrm{BII} / \mathrm{o}: 2,44)$. Or, ce qui intéresse Xenakis est d'offrir différentes longueurs d'ondes que les dessinateurs de l'atelier peuvent utiliser à volonté par unité ou en combinaison, selon la longueur de la façade qu'ils doivent vitrer. D'où l'intérêt de ce tableau qui accompagne le brevet de pans de verre "ondulatoires»; il fournit un indicateur des longueurs globales des ondes.

C'est d'ailleurs grâce à cette constatation que nous avons pu déchiffrer les formes d'onde que nous avons exposées auparavant. Sur la segmentation de la façade du plan $\mathrm{N}^{\circ} 31598$ [fig.18], lorsqu'il s'agit d'un segment court - qui contient une seule onde - la réponse est relativement facile à deviner; il faut se tourner vers les ondes dont la longueur se rapproche de la valeur du segment ${ }^{36}$. Cependant, dans le cas d'un segment plus grand, qui semble conclure deux ou plusieurs ondes, nous avons procédé au raisonnement suivant: parmi toutes les ondes présentées sur le tableau $\mathrm{N}^{\circ} 6030$ du brevet, nous cherchons celles dont l'addition de longueurs se rapproche le plus possible des valeurs de la segmentation mentionnée ci-dessus. Ainsi pour le premier étage, segment 8,71-qui a été le plus difficile à interpréter étant donné que plusieurs distances ont été assemblées pour former les ouvertures de la cuisine - on peut se rapprocher de la longueur globale 8,71 seulement par la juxtaposition de $1 / 2$ longueur de $\mathrm{A}^{\mathrm{I}} / \mathrm{o}$ et de 1 longueur de $\mathrm{A}^{\%} / 1^{37}$. Or, le compartimentage de la façade permet à Xenakis de circonscrire les régions à l'intérieur desquelles il devrait poser des formes d'ondes dont les longueurs globales se rapprochent le plus possible des valeurs de la segmentation.

Cette pratique de segmentation apporte à Xenakis la solution d'un problème, qu'il serait autrement impossible d'aborder avec les moyens dont il disposait à l'époque: sur une droite, qui représente la longueur de la façade, comment distribuer les différentes longueurs d'ondes, qui sont des ensembles de pans de verre? N'ayant pas encore en main la théorie des probabilités (il va l'appliquer dans sa musique l'année suivante), Xenakis répond à ce dilemme avec une méthode beaucoup plus élémentaire: il découpe la longueur globale de la façade en plus petites parties, de telle sorte que la segmentation coïncide avec la juxtaposition de la longueur d'une ou de plusieurs formes d'ondes. En d'autres mots, il rétrécit le nombre de combinaisons possibles des ondes - et il allège ainsi son problème de choix - en raccourcissant littéralement la longueur à l'intérieur de laquelle il doit les distribuer.

36 Une correspondance absolue est exclue a priori puisque les membrures de béton supplémentaires, qui sont ajoutées afin de construire les parties denses, augmentent la longueur de l'onde initialement choisie.

37 Nous avons effectué toutes les combinaisons possibles des ondes présentées dans le tableau de brevet. 


\section{b) La propriété additive de Modulor et la série Fibonacci}

Nous avons montré que les parties «denses» de la façade ouest sont produites par l'assemblage de petits pans de verre à la place d'une ou de plusieurs grandes distances de l'onde initialement choisie. Ainsi, soit l'architecte «divise» les grandes distances d'une onde en plus petites (ex. troisième ou deuxième étage, segment $8,36 \mathrm{~m}$, deuxième tronçon onde $\mathrm{B}^{\mathrm{VI} / 2}$ éléments $0,7 \mathrm{O} \& 1,40$ ); soit il passe par l'assemblage - plus rare - de petits éléments pour construire des pans de verre plus grands (ex. troisième étage, segment $13,16 \mathrm{~m}$, premier tronçon, élément 1,83). Or, chaque partie dense a une longueur totale (membrures de béton compris) qui équivaut à une ou à plusieurs distances tirées des séries du Modulor. En produisant des grandes densités à l'intérieur des ondes, Xenakis contrôle «la durée» de chaque densité, c'est-à-dire la longueur sur laquelle la densité s'étale, comme son texte nous le rappelle ${ }^{38}$.

À la même époque, Xenakis bâtit aussi un rythme "additif» pour son œuvre Metastaseis 39 . Dans son texte "Metastaseis et le Modulor", à la fin du Modulor 2 de Le Corbusier, Xenakis parle de la propriété additive et explique le rôle qu'elle joue dans la composition des Metastaseis :

Une des applications essentielles des «Metastassis» dans cet ordre d'idées est que les six intervalles algébriques ${ }^{40}$ et tempérés de la gamme de douze sons sont émis dans des durées proportionnelles aux rapports de fréquences. D’où des gammes de six durées ${ }^{41}$ accompagnent l'émission des intervalles. La suite des intervalles tempérés est une progression géométrique. Les durées le seront également. Par ailleurs, la durée a la propriété additive. Une durée peut être additionnée à une autre et leur somme est sentie comme telle. D’où une nécessite naturelle d'avoir des gammes de durées qui puissent s'additionner ${ }^{42}$ dans le sens défini plus haut. Parmi toutes les progressions géométriques, il n'y en a qu'une seule dont les termes jouissent de cette propriété additive. C’est la progression de la Section d'Or. Voilà de quelle façon l'idée du Modulor a créé une liaison étroite de structure entre le temps et les sons. (Xenakis 1955a, p. 340-344, nous soulignons).

Dans Metastaseis, Xenakis utilise la propriété additive et fait appel à la série Fibonacci 43 et non au Modulor afin de mettre en place «la durée différentielle» (Barthel-Calvet 2003, p. 131-136). Comme on l'a vu, une durée différentielle, par exemple, de valeur de 0,05 de la noire, correspond à l'écart entre une double croche normale (égale à 0,25 noire) et une double croche de quintolet (égale à

38 Xenakis 2006, p. 116, nous soulignons : «Donc la solution est de juxtaposer des parties denses (beaucoup de montants en béton armé) à des raréfiées. Ici, naturellement, il faut définir les degrés de densité et leurs longueurs (durées).»

39 Pour la différence entre rythme additif et divisionnel, voir Barthel-Calvet 2000, p. 95.

40 Qui apparemment veut dire $+1,+-2,+-3,+-4,+-5,+-6$.

41 Si on tient au tableau $\psi$ (Xenakis dans Barthel-Calvet, 2003, p. 164), ces durées sont les : 0,05; 0,$08 ; 0,13 ; 0,21 ; 0,34 ; 0,55 ; 0,89 ; 1,44 ; 2,33$.

42 Par exemple : $0,05+0,08=0,13$

43 La suite Fibonacci $(0,1,1,2,3,5,8,13$, 21, etc.) est une suite géométrique de nombres entiers dont chaque terme est la somme des deux termes qui le précèdent : $f n=f n-1+f n-2$. Le ratio entre deux termes consécutifs est constant et il se rapproche du coefficient de la section d'or $(1,618 \ldots)$. 
0,20 noire). Ainsi, la durée-différentielle, qui gère «en arrière-plan» les rapports de durées des sons des différentes lignes mélodiques, devient un nouvel élément «rythmique» pour Xenakis. Elle se fonde sur la propriété additive qui caractérise par nature les durées ainsi que les termes de la suite Fibonacci.

En architecture, la substitution des grandes distances - lors de la création des parties denses - par un assemblage de petits pans de verre, se fonde sur la propriété additive, qui est inhérente aux séries du Modulor; l'addition de deux termes successifs de ces séries donne le terme suivant $(70+43=1,13)$. Ainsi une distance de verre quelconque peut être remplacée par la somme des plus petites valeurs, auxquelles à leur tour peuvent se substituer la somme des valeurs encore plus petites et ainsi de suite. Laddition de petites valeurs de pans de verre aboutit à la construction des parties «denses» de la façade. Dans ce cas aussi, la propriété additive se trouve derrière un élément «rythmique» de la façade, les longueurs globales des parties denses. Mais à l'évidence, ce qui se transpose de la musique en architecture (ou l'inverse) est l'idée d'un rythme «additif» et en aucun cas les valeurs elles-mêmes (suite Fibonacci contre les séries du Modulor).

\section{c) La notion de densité}

Quel est le rapport entre la définition que Xenakis réserve à la notion de densité dans le cadre de sa musique et l'emploi de cette même notion lors de l'organisation des pans de verre «ondulatoires» du couvent?

Dans sa musique instrumentale, Xenakis fait «officiellement» appel à la notion de densité pour la première fois avec l'intention d'organiser par des lois de probabilités les paramètres (hauteur, durée, vitesse) d'un grand nombre de sons («masses sonores»). En 1956, Xenakis (1971, p. 9-15), dans le texte «Théorie des probabilités et composition musicale», utilise d'abord la densité comme un élément rythmique qui gère la répartition des durées.

Dans ce cadre, (Barthel-Calvet 200o, p. 301-327 et p. 385-411) la densité (linéaire) permet au compositeur de déterminer la répartition des dates d'occurrence des événements sonores. La date d'occurrence d'un son est l'instant d'attaque d'un son, dont la nécessité est comprise en lien avec la durée différentielle. En effet, Xenakis, en s'intéressant aux décalages entre les durées de deux sons et non pas aux durées propres des sons, a besoin de définir le moment où les deux sons apparaissent. Si le temps est considéré comme une ligne droite, les dates d'apparition des sons correspondent à des points sur cette ligne; l'intervalle entre deux points donne leur «durée différentielle». Ainsi,

Parmi toutes les successions possibles de points, laquelle est à choisir? Ainsi posée, la question n'a pas de sens. Je désigne une moyenne de points sur une longueur donnée. La question devient: «Étant donné cette moyenne de points, entre quelles limites les segments ainsi créés peuventils varier?» La formule qui découle des raisonnements des probabilités continues et qui donne les probabilités pour toutes ces longueurs possibles, lorsqu'on connaît la moyenne des points placés au hasard sur une droite, est: 


$$
\mathrm{Px}=\delta \varepsilon^{-\delta x} \mathrm{dx}
$$

dans laquelle $\delta$ est la densité linéaire des points et $\chi$ la longueur d'un segment quelconque. (Xenakis 1971, p. 10).

Or, c'est à partir de la densité moyenne des points sur une ligne (point = la date d'occurrence d'un son) qu'il peut calculer la probabilité que telle ou telle longueur entre deux points (durée différentielle) surgisse. Dans le cas d'une densité faible, les dates d'occurrence des sons sont relativement éloignées; dans le cas inverse, celui d'une densité importante, les événements se rapprochent. C'est en ce sens que la densité linéaire devient un facteur rythmique déterminant (Barthel-Calvet 200o, p. 301-327 et p. 385-411).

Pendant la même période, les premières œuvres de musique «électroacoustique» du compositeur voient le jour. Xenakis semble entamer l'étude de sa pièce Diamorphoses (1957-58), en janvier 1957 selon Makis Solomos (2011); cette date se situe juste quelques mois après la version finale de la façade ouest du couvent (plan $\left.\mathrm{N}^{\circ} 2046,20 / 11 / 56\right)$. Un des enjeux compositionnels de cette œuvre musicale est la perception logarithmique de la densité des sons de cloches. Solomos (2011, p. 22) reproduit, dans son analyse, une lettre inédite de Xenakis qui explique ce travail sur la densité:

Avec les cloches j'ai fait une double étude: a) combien de sons de courte durée nous pouvons percevoir pendant un court intervalle de temps $\Delta \mathrm{t}$ (jusqu'à 5 ; après il nous est impossible de calculer); b) la sensation de densité (nombre de sons/sec) et la loi qui lie la sensation et la densité. Pour le b) j'ai trouvé que la loi est logarithmique avec une base entre 2 et 3 , et c'est pour cela que j'ai choisi le nombre $e=2,718^{44}$.

Pour Diamorphoses, Xenakis fait appel à la définition mathématique de la densité (nombre d'éléments par unité métrique); il cherche aussi à dégager une loi qui lie la sensation acoustique d'une partie riche en différents sons et le nombre de sons par seconde qui caractérise une telle sensation.

Pendant l'élaboration des façades du couvent (1956-57), la notion de densité garde sa définition mathématique première: nombre de pans de verre par unité métrique. Elle n'a pas été calculée, selon notre raisonnement, à partir des lois de probabilités. Une grande densité contient un grand nombre de petites distances en verre qui est produit par le «morcellement» des grandes distances que l'onde contient. D'ailleurs, le jeu entre les parties denses et raréfiées a été géré par une opposition des dynamiques $(f-f f$, crescendo - decrescendo), comme le plan [fig.16], désormais perdu, incite à le penser.

Mais même l'emploi que Xenakis réserve à la notion de densité dans le cadre architectural, contient en germe l'aspect qu'il va essayer d'approfondir dans ses compositions l'année suivante. Dans sa musique instrumentale, la notion

44 Nous traduisons : «With the bells I did a double study: a) how many short sounds can we perceive in a short delta $t$ (up to 5 , then we lose the counting ability); b) the sensation of density (numbers of sounds / sec) and the law that binds sensation and density. For b) I found the law is logarithmic with a base between 2 and 3, this is why I choose the number e $=2,718$. A consequence of this finding was the formula (p. 136 in Formalized Music) for the ST instrumental music series [...].» 
de densité aide Xenakis à calculer une distribution irrégulière de la «durée différentielle», en faisant appel aux théories des probabilités. Il procède ainsi à une détermination statistique des paramètres (date d'apparition, hauteur) qui définissent un phénomène sonore. Mais il est conscient que tant dans le champ visuel qu'auditif, nous procédons à des jugements d'ordre statistique:

La densité est une perception macroscopique, un calcul inconscient que nous faisons instantanément aussi bien dans le cas visuel qu'auditif. Cette faculté procède de notre construction mentale, qui est aussi statistique. Nous faisons de l'analyse (synthèse) statistique sans le savoir. (Xenakis 1984, dans Kanach 2006, p. 113).

Quand les densités se juxtaposent linéairement sur chaque étage et verticalement sur l'ensemble des étages, elles sont d'abord perçues statistiquement, cela signifie qu'elles sont perçues à travers un classement élémentaire: «densités grandes» contre «densités faibles». La théorie des probabilités sera un autre moyen, plus savant sans doute, d'établir, au cours des années suivantes, un tel classement dans le domaine de la musique.

L'application de la notion de densité au sein de l'architecture semble être ici un préambule à l'étude approfondie que Xenakis réserva à cette notion dans ses compositions musicales. Sa pratique d'architecte devient ainsi le laboratoire qui permet à Xenakis d'expérimenter des notions, avant de les approfondir dans ses œuvres musicales.

\section{d) Lois d'enchaînement des séries}

Nous avons vu l'enchaînement linéaire des différentes formes d'ondes sur la façade ouest du couvent. Pour les compositeurs sérialistes de l'époque, l'enchaînement des séries de douze notes a aussi été une de leurs préoccupations majeures. Pour Pierre Boulez, par exemple, les notes-pivot (notes communes entre deux séries) et l'enchaînement des parties semblables des séries (ambiguité harmonique) étaient des possibilités de transition d'une série à l'autre ${ }^{45}$. Pouvons-nous dégager des liens de parenté entre l'enchaînement des séries que Xenakis envisage dans la partie médiane des Metastaseis et la succession des formes d'ondes sur la façade du couvent?

Dans la partie médiane des Metastaseis, comme nous l'avons vu, Xenakis voulait radicaliser les transformations d'une série de sons (droite, miroir et ses rétrogrades) en introduisant les procédés de permutations. Pour les 24 permutations de quatre sons des mesures 104-150, il prévoit des lois élémentaires pour passer d'un arrangement des quatre notes au suivant. Mais que se passe-t-il

45 Boulez 1995, p. 272, nous soulignons : «Comment enchaîner les séries les unes aux autres est un des problèmes les plus naïfs, mais apparemment les plus délicats de la technique sérielle. S'il n'y a pas, en effet, une logique générale de succession, on aboutirait au paradoxe inutile d'une organisation infrastructurelle hiérarchique, les superstructures étant livrées à une indolente anarchie, où l'empirisme - pas toujours de bon aloi, il est à redouter - tâtonne et éprouve quelques recettes. Nous avons déjà signalé les notes-pivots : ce moyen nous semble un peu rudimentaire et capricant. Faire voisiner des séries à régions semblables est infiniment plus satisfaisant, puisque l'ambiguïté harmonique joue de ce fait un très grand rôle; cela suppose cependant des séries à propriétés remarquables, et implique de jouer à peu près toujours sur les mêmes propriétés.» 
quand le nombre de permutations d'éléments est nettement plus grand? Comment Xenakis envisage-t-il le passage de l'un à l'autre?

Dans les mesures 202-309 des Metastaseis, Xenakis s'efforce de former des séries en tenant compte des intervalles entre les notes. En fait, il cherche à produire une méthode de classement des millions de séries qui sont produites par les permutations directes des 12 sons chromatiques $(12 !=479.001 .600)$. Ainsi chacun de ces 12 sons, à l'intérieur d'une octave, forme avec un son quelconque défini comme origine, un intervalle qui peut prendre les valeurs suivantes $+/-0$, $+/-1,+/-2,+/-3,+/-4,+/-5,+/-6$ (avec $+/-1=1$ demi-ton). À l'intérieur de la masse des presque 500 millions de séries, Xenakis applique aux intervalles qui séparent les notes de chaque série les lois sérielles d'exhaustivité et de non répétition (Xenakis 1954, dans Barthel-Calvet 2003, p. 172).

Ainsi, une série de 12 sons est divisée en deux tronçons égaux, dont chacun doit présenter une fois et une seule les intervalles $1,2,3,4,5,6$. Ce critère lui permet de choisir seulement 195 permutations de séries parmi les millions possibles. Ici, Xenakis cherche à souligner une raison d'ordre musical dans la succession des intervalles pour éviter l'arbitraire des permutations. Il forme ainsi huit familles de «demi-séries mères» selon la «tension» entre les intervalles ${ }^{46}$.

$$
\begin{aligned}
& \alpha: 345612 \text { \&: } 216345 \\
& \beta: 345216 \zeta: 612543 \\
& \gamma: 543612 \text { Z: } 216543 \\
& \delta: 543216 \mathrm{H}: 612345
\end{aligned}
$$

L'ensemble des 195 séries d'intervalles sont en fait les 23 ramifications ( $\alpha^{\prime}, \alpha^{\prime \prime}, \beta^{\prime}$, $\beta$ ", etc.) de ces 8 demi-séries mères [fig. 23] (Barthel-Calvet 2011, p. 8). Parmi celles-ci, pour les organiser davantage, Xenakis dégage des familles de séries, fondées sur les ressemblances ou les égalités entre les intervalles de chacun des deux tronçons [fig.24].

Ainsi selon Barthel-Calvet (2003, p. 158):

- il y a 54 séries d'intervalles, prises par deux, dont les deux tronçons sont identiques mais ont changé de place, comme par exemple celles-ci:

$\varepsilon 9 \mathrm{~F}=+3-1+4-5+6+2 /-5+4-3+-1+2$

$-\gamma 7 \mathrm{~F}=-5+4-3-6-1+2 /+3-1+4-5-6+2$.

- il y a 16 séries d'intervalles, prises par deux, dont un seul des deux tronçons est identique. Dans le texte, Xenakis fait apparaître 8 "égalités remarquables» de cette nature, dont en voici une ici :

$\varepsilon^{\prime \prime \prime} 4=-1-2-6-5+4+3 /-5+4-3+6-1+2$

$-\delta^{\prime \prime} 1=-5-4+3-2+1-6 /-1-2-6-5+4+3$.

46 Xenakis 1954, dans Barthel-Calvet 2003, p. 172. Deux sons relativement proches en hauteur produisent un intervalle qui est moins tendu que deux sons dont la hauteur est plus éloignée. Xenakis explique: «on admet que 2 est moins tendu que 1 et 6 ». 
Les 16 séries qui présentent, prises par deux, les huit «égalités remarquables», n’appartiennent pas aux mêmes huit familles de "demi-séries mères». Or, lorsqu'il fait succéder des séries qui présentent des "égalités remarquables", Xenakis passe d'une famille à l'autre. Barthel-Calvet (2011, p. 10) montre comment tous ces critères d'égalité jouent un rôle dans l'enchaînement des 195 séries d'intervalles dans les mesures 202-308.

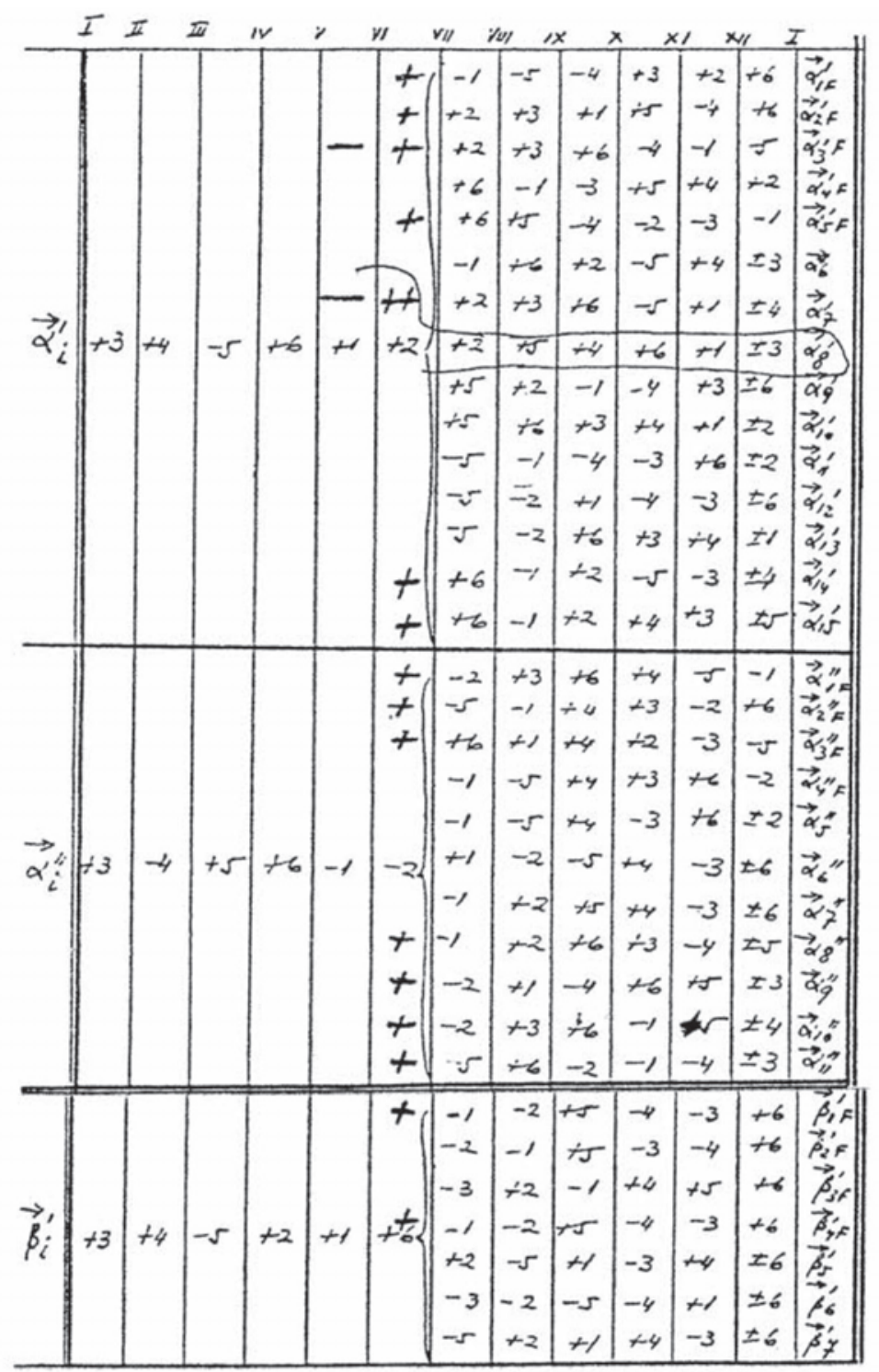

Figure 23. Exemple parmi les 195 séries d'intervalles, par Xenakis, publié dans Barthel-Calvet, 2003, p.175. Avec l'aimable autorisation de Barthel-Calvet. 


$$
\begin{aligned}
& \vec{\alpha}_{4 \mathrm{~F}}^{\prime \prime}=-\overrightarrow{\mathrm{z}}_{2 \mathrm{~F}}^{\prime \prime}, \vec{\alpha}_{1 \mathrm{~F}}^{\prime}=\overrightarrow{\mathrm{Z}}_{4 \mathrm{~F}}^{\prime}, \vec{\alpha}_{4 \mathrm{~F}}^{\prime}=\overrightarrow{\mathrm{z}}_{2 \mathrm{~F}}^{\prime}, \bar{\alpha}_{2 \mathrm{~F}}^{\prime \prime}=-\overrightarrow{\mathrm{z}}_{3 \mathrm{~F}}^{\prime \prime} \text {, } \\
& \bar{\alpha}_{4 \mathrm{~F}}^{\prime \prime}=-\overrightarrow{\mathrm{Z}}_{2 \mathrm{~F}}^{\prime \prime}, \vec{\alpha}_{3 \mathrm{~F}}^{\prime}=\overrightarrow{\mathrm{Z}}_{3 \mathrm{~F}}^{\prime}, \vec{\alpha}_{5 \mathrm{~F}}^{\prime}=\overrightarrow{\mathrm{Z}}_{1 \mathrm{~F}}^{\prime} \text {. } \\
& \vec{\beta}_{4 \mathrm{~F}}^{\prime}=\vec{\zeta}_{\mathrm{IF}}^{\prime \prime}, \vec{\beta}_{3 \mathrm{~F}}^{\prime \prime}=-\vec{\zeta}_{\mathrm{IF}}^{\prime \prime \prime}, \vec{\beta}_{1 \mathrm{~F}}^{\prime \prime \prime}=\vec{\zeta}_{4 \mathrm{~F}}^{\prime}, \vec{\beta}_{3 \mathrm{~F}}^{-\prime \prime}=\vec{\zeta}_{5 \mathrm{~F}}^{\prime}, \bar{\beta}_{1 \mathrm{~F}}^{\prime \prime}=-\vec{\zeta}_{3 \mathrm{~F}}^{\prime \prime \prime} \text {, } \\
& \vec{\beta}_{4 \mathrm{~F}}^{\prime \prime \prime}=-\vec{\zeta}_{4 \mathrm{~F}}^{\prime \prime \prime}, \bar{\beta}_{2 \mathrm{~F}}^{\prime \prime \prime}=\vec{\zeta}_{1 \mathrm{~F}}^{\prime}, \vec{\beta}_{4 \mathrm{~F}}^{-\prime \prime}=\vec{\zeta}_{2 \mathrm{~F}}^{\prime} \\
& \overline{\mathrm{H}}_{1 \mathrm{~F}}^{\prime \prime \prime}=\vec{\delta}_{1 \mathrm{~F}}^{\prime}, \tilde{\mathrm{H}}_{1 \mathrm{~F}}^{\prime}=\vec{\delta}_{1 \mathrm{~F}}^{\prime \prime \prime}, \overline{\mathrm{H}}_{3 \mathrm{~F}}^{\prime \prime}=-\bar{\delta}_{5 \mathrm{~F}}^{\prime \prime} \text {, } \\
& \bar{\varepsilon}_{1 \mathrm{~F}}^{\prime}=-\bar{\gamma}_{6 \mathrm{~F}}, \bar{\varepsilon}_{2 \mathrm{~F}}^{\prime \prime \prime}=-\dot{\gamma}_{2 \mathrm{~F}}^{\prime \prime \prime}, \bar{\varepsilon}_{3 \mathrm{~F}}^{\prime}=-\vec{\gamma}_{7 \mathrm{~F}}^{\prime \prime \prime}, \bar{\varepsilon}_{1 \mathrm{~F}}^{\prime \prime}=\vec{\gamma}_{7 \mathrm{~F}}^{\prime}, \bar{\varepsilon}_{2 \mathrm{~F}}^{\prime \prime \prime}=-\vec{\gamma}_{6 \mathrm{~F}}^{\prime \prime} \text {, } \\
& \bar{\varepsilon}_{3 \mathrm{~F}}^{\prime \prime \prime}=-\vec{\gamma}_{9 \mathrm{~F}}^{\prime \prime}, \bar{\varepsilon}_{9 \mathrm{~F}}^{\prime \prime \prime}=-\vec{\gamma}_{7 \mathrm{~F}}^{\prime}, \bar{\varepsilon}_{10 \mathrm{~F}}^{\prime \prime \prime}=-\vec{\gamma}_{6 \mathrm{~F}}^{\prime}, \bar{\varepsilon}_{11 \mathrm{~F}}^{\prime \prime \prime}=-\vec{\gamma}_{9 \mathrm{~F}}^{\prime}
\end{aligned}
$$

Figure 24. Les familles de séries de notes pour les mes. 202-309 de Metastaseis fondées sur les ressemblances ou les égalités entre les intervalles, par Xenakis «Metastassis-Analyse», in Barthel-Calvet, 2003, p. 174. Avec l'aimable autorisation de Barthel-Calvet.

Du côté de l'architecture maintenant, nous avons constaté qu'à l'intérieur des formes d'ondes, les grandes distances se «morcellent» en plus petites pour engendrer les parties denses. Une étude attentive des toutes les ondes transformées en "parties denses» montrera que les densités, une fois produites, changent de place pour se placer aux limites de l'onde, afin d'assurer la continuité avec l'onde suivante; les éléments «non-transformés» de l'onde initiale se mettent à l'autre bout. Ainsi une chaîne de petites distances de verre - produisant une "densité» - se met dans la continuité de l'ordre croissant/décroissant des distances de verre de l'onde suivante. Nous suivons cette règle élémentaire d'«enchevêtrement», d'une homogénéité de matériau, par exemple pour le deuxième étage: [fig.25]. Selon nous, la création de grandes densités de pans de verre aux limites des ondes permet à Xenakis d'avoir une transition «ondulatoire» fluide ou bien une saccade nette entre le déploiement de deux ondes successives.

Nous constatons aussi que l'enchaînement entre deux ondes successives est réalisé par une distance commune aux deux ondes (deuxième étage, distance 0,53 commune entre l'onde $\mathrm{B}^{\mathrm{VI}} / 2$, segment 8,14 et l'onde $\mathrm{B}^{\mathrm{VI}} / 2$, segment 4,33). Cette distance-pivot respecte d'une certaine façon la règle sérielle «de non répétition ", en faisant apparaître une seule fois la distance en question dans l'enchaînement des deux ondes, qui contiennent toutes les deux dans leur forme originale cette même longueur de verre.

Xenakis joue avec ces deux règles: distances-pivot entre deux séries successives et enchaînement des parties denses avec des ondes présentant une "homogénéité» de matériel à leurs extrémités; il s'appuie sur ces deux pratiques compositionnelles pour enchaîner les différentes ondes linéairement sur la façade du couvent. D'ailleurs ces règles semblent résoudre des problèmes similaires lors de l'élaboration des façades du couvent: 


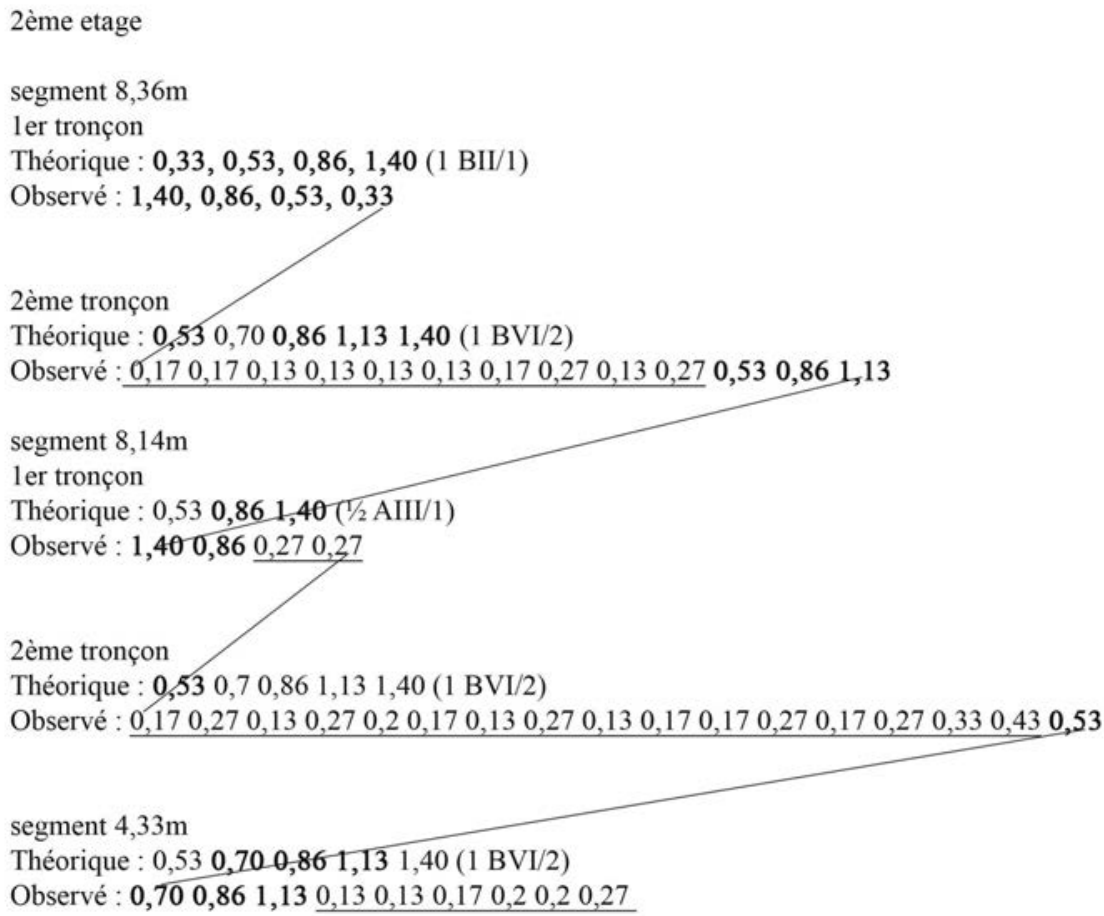

Figure 25. Les densités, une fois produites, changent de place pour se placer aux limites de l'onde, afin d'assurer la continuité avec l'onde suivante; les éléments «non-transformés» de l'onde initiale se mettent à l'autre bout. Graphique établi par Elisavet Kiourtsoglou.

Mais, de plus, un autre problème surgit, c'est celui du passage d'une densité à une autre, en progression continue, ou brutalement, par saccades. Le problème de la continuité dans la transition, ainsi que sa vitesse ou sa forme, jouent des rôles fondamentaux en esthétique musicale ou en arts plastiques et en architecture. (Xenakis 1984 dans Kanach 2006, p. 116).

Les réponses que nous fournissons ici montrent qu'une fois les idées principales choisies et concrétisées (onde-série des éléments, densité, compartimentage de la façade, rythme additif), l'enchaînement linéaire et vertical des éléments repose sur des pratiques plutôt ordinaires pour un compositeur, mais fortement originales pour un architecte.

\section{Conclusion}

Xenakis, pour l'élaboration des pans de verre «ondulatoires» dans le Couvent de la Tourette, fait appel à ses recherches musicales. Il s'agit d'un transfert de notions (comme la densité et le compartimentage en section d'Or), qui ne sont pas purement musicales: cela permet en effet à Xenakis d'instaurer un laboratoire où ses avancements en musique l'aident dans l'architecture et vice-versa. D’ailleurs, la propriété additive des valeurs du Modulor à laquelle il fait appel 
lorsqu'il construit des densités et des raretés de pans de verre sur les façades du couvent a été déjà mise en valeur pour la «durée différentielle» dans Metastaseis, et elle lui permettra plus tard de jongler avec la densité des sons sur la bande magnétique ou les dates d'occurrence des glissandi dans Pithoprakta. De surcroit, certaines pratiques compositionnelles ordinaires, en provenance d'un vocabulaire sériel (note-pivot, matériau homogène), lorsqu'elles répondent aux questions architecturales, y apportent de l'originalité: c'est le changement de contexte qui est maintenant déclencheur de nouveauté et non les notions en elles-mêmes. La seule fois que l'outil mathématique passe directement de la musique en architecture (la combinatoire des Metastaseis utilisée pour la distribution des pans de verre des façades intérieures), il doit se rajuster pour répondre aux exigences de l'art de bâtir.

Comme c'est le cas toujours lors d'une analogie, le transfert se fonde sur l'idée abstraite en commun et non pas sur la transposition de son expression explicite; ce sont ces moments libérateurs, très importants à l'émergence de Xenakis comme compositeur et architecte, où il se permet de franchir les frontières d'un domaine à l'autre avec un simple «et si c'était?». L'analogie, notion qui puise ses origines dans la pensée philosophique-mathématique présocratique, devient chez Xenakis le point central de sa créativité.

\section{Archives}

Collection famille Xenakis, X(A): Tourette

Fondation Le Corbusier

\section{RÉFÉRENCES}

Baltensperger, André. 1996. Iannis Xenakis, Komposition im Spannungsfeld der Architektur und Musik. Basel: Haupt Verlag.

Barthel-Calvet, Anne-Sylvie. 2000. «Le rythme dans l'œuvre et la pensée de Iannis Xenakis.» Thèse de doctorat, EHESS, Paris.

-2003. «METASTASSIS-Analyse: Un texte inédit de Iannis Xenakis sur Metastasis». Revue de Musicologie 89, no: 129-187.

-2009. «De l'ubiquité poétique dans l'œuvre de Iannis Xenakis — Espace, Temps, Musique, Architecture». Intersections: Revue canadienne de musique $29, \mathrm{n}^{\circ} 2: 9-51$.

-2011. "Xenakis et le sérialisme: l'apport d'une analyse génétique de Metastasis». Intersections: revue canadienne de musique 31, $\mathrm{n}^{\circ} 2: 3-21$.

Bridoux-Michel, Séverine. 2006. "Architecture et musique: Croisements de pensées après 1950 ». Thèse de doctorat, Université de Lille 3.

Boulez, Pierre, 1955. «Éventuellement». Points de repère. Tome I, Imaginer, sous la dir. de Jean-Jacques Nattiez et Sophie Galaise; exemples musicaux identifiés par Robert Piencikowski. Paris: Christian Bourgois.

Delalande, François. 1997. «Il faut être constamment un immigré»: Entretiens avec Xenakis. Paris: INA \& Buchet/Chastel.

Gibson, Benoitt. 2011. The instrumental music of Iannis Xenakis. New York: Pendragon Press.

Ferro et al. 1987. Le Corbusier, Le Couvent de la Tourette. Marseille: Éditions Parenthèses. 
Kanach, Sharon. 2006. Iannis Xenakis: Musique de l'architecture. Marseille: Éditions Parenthèses.

Kiourtsoglou, Elisavet. 2017. "An Architect Draws Sound and Light: New Perspectives on Iannis Xenakis's Diatope and La Legènde d'Eer (1978)». Computer Music Journal 41, ${ }^{\circ}$ 4, The MIT Press pp. 8-31.

.2016. «Le travail de l'analogie dans la musique et l'architecture de Iannis Xenakis». Thèse de Doctorat, Université Paris 8.

Le Corbusier. 1949. Le Modulor. Essai sur une mesure harmonique à l'échelle humaine applicable universellement à l'architecture et à la mécanique. Paris: Éditions de l'Architecture d'Aujourd'hui. (Réédition fascimilée Basel: Birkhaüser, 2011).

__ 1955. Modulor 2, 1955 (La parole aux usagers), suite de "Le Modulor» "1948". Basel: ed. Birkhaüser \& Paris: Fondation Le Corbusier. (Réimpression de l'édition originale, 2011).

Matossian, Nouritsa. 1981. Iannis Xenakis. Paris: Fayard.

Solomos, Makis. 2011. «Xenakis' first composition in musique concrète: Diamorphoses ». On-line proceedings of Xenakis International Symposium CCMC, Goldsmiths, University of London Southbank Centre 1-3 April 2011. http://www.gold.ac.uk/media/migrated/media/goldsmiths/departments/ academicdepartments/music/pdf/Keynote-III-Makis-Solomos.pdf

Sterken, Sven, 2003-4. «Iannis Xenakis: ingénieur et architecte». Thèse de doctorat, Université de Gent.

Xenakis, Iannis. 1954. "METASTASSIS-Analyse» publié dans Barthel-Calvet 2003, p.162-87.

. 1955a. Sans titre. Le Corbusier 1955, p.340-344.

- 1955b. "Note relative aux pans de verre dénommés "ondulatoires" ». Kanach 2006, p.77.

—_ 1956. "Wahrscheinlichkeitstheorie und Musik», Gravesaner Blätter $\mathrm{n}^{\circ} 6: 28$-34. La version française, «Théorie des probabilités et composition musicale», dans Xenakis 1971, p. 9-15 à laquelle nous renvoyons.

_.1963. Préface de la partition de MetastaseisB (1953-54). Boosey \& Hawkes. .1965. «Cribles». Xenakis. Kéleütha, p. 75-87. Préface et notes Benoit Gibson. Paris: L'Arche.

__ 1971. Musique/Architecture. Tournai: Casterman.

- 1984. "The Monastery of La Tourette». A Series in Garland Architectural Archives. Le Corbusier. La Tourette and Other Building Projects 1955-57, sous la dir. de Allen Brooks. London-New York-Paris: Garland Publishing Inc. / Fondation Le Corbusier, p. ix-xviii. (La version française, «Le monastère de la Tourette», Kanach 2006, p.105-119 à laquelle que nous renvoyons).

\section{RÉSUMÉ}

Les pans de verre «ondulatoires» du Couvent de la Tourette (1953-1961) - une distribution irrégulière de pans de verre de longueurs graduellement variables - se présentent comme une nouvelle version de l'ancienne analogie entre musique et architecture; 
c'est Iannis Xenakis, qui travaillait comme ingénieur et architecte dans l'atelier Le Corbusier, qui les a conçus en faisant appel à ses recherches musicales. Dans cet article, nous proposons une hypothèse pour déchiffrer leur conception et leur rapport avec son travail de compositeur, restés longtemps peu détaillés, en interprétant des éléments tirés des archives.

\section{ABSTRACT}

The "undulatory" glass pieces of the Tourette Convent (1953-1961) - constituted of an irregular distribution of glass pieces of various lengths - appears to be a new expression of the traditional and long-lasting analogy between music and architecture. Its creator, Iannis Xenakis, created them drawing from his research in music, when he was working as an engineer and architect at the Le Corbusier workshop. On the basis of archival documents, this essay develops an hypothetical method to decipher their conception and link to his work as a composer, aspects which have not been examined for a long time.

\section{BIOGRAPHIE}

Elisavet Kiourtsoglou (1981) est architecte-ingénieur diplômée de l'Université Polytechnique de Volos (2005). Elle détient un post-master en histoire et théorie d'architecture de l'École Polytechnique d'Athènes (2008). En 2016, elle a soutenu sa thèse de doctorat sur l'analogie entre musique et architecture dans l'œuvre de Iannis Xenakis (Paris 8), recherche qui a été financée par plusieurs fondations privées et publiques. Elle a présenté ses recherches dans plusieurs colloques internationaux en France et à l'étranger : 4oth International Computer Music Conference (ICMC), Université Paris 8, European University (Cyprus). En parallèle, elle alimente sa réflexion et sa pratique d'architecte par des performances, des installations et des écrits sur les thèmes de la mémoire et du rapport du corps à l'espace. Actuellement, elle est enseignante à l'Ecole Nationale Supérieure d'Architecture de Strasbourg. 